\title{
Title: Multifactorial chromatin regulatory landscapes at single cell resolution
}

Authors: Michael P. Meers ${ }^{1}$, Derek H. Janssens ${ }^{1}$, Steven Henikoff ${ }^{1,2}$

Affiliations: ${ }^{1}$ Basic Sciences Division, Fred Hutchinson Cancer Research Center, Seattle, WA, USA; ${ }^{2}$ Howard Hughes Medical Institute, Chevy Chase, MD, USA

Abstract: Chromatin profiling at locus resolution uncovers gene regulatory features that define cell types and developmental trajectories, but it remains challenging to map and compare distinct chromatin-associated proteins within the same sample. Here we describe a scalable antibody barcoding approach for profiling multiple chromatin features simultaneously in the same individual cells, Multiple Target Identification by Tagmentation (MulTI-Tag). MulTI-Tag is optimized to retain high sensitivity and specificity of enrichment for multiple chromatin targets in the same assay. We use MulTI-Tag to resolve distinct cell types using multiple chromatin features on a commercial single-cell platform, and to distinguish unique, coordinated patterns of active and repressive element regulatory usage in the same individual cells. Multifactorial profiling allows us to detect novel associations between histone marks in single cells and holds promise for comprehensively characterizing cell-specific gene regulatory landscapes in development and disease. 


\section{Main Text}

Single-cell sequencing methods for ascertaining cell type-associated molecular characteristics by profiling the transcriptome ${ }^{1-3}$, proteome $e^{4-6}$, methylome ${ }^{7,8}$, and accessible chromatin landscape $\mathrm{e}^{9,10}$, in isolation or in "Multimodal" combinations ${ }^{11-15}$, have advanced rapidly in recent years. More recently, methods for profiling the genomic localizations of proteins associated with the epigenome, including Tn5 transposasebased Cleavage Under Targets \& Tagmentation (CUT\&Tag) ${ }^{16,17}$, have been adapted for single cell profiling, overcoming the sparse incidence of such proteins in comparison with other molecular markers. The combinatorial nature of epigenome protein binding and localization ${ }^{18-20}$ presents the intriguing possibility that a method for profiling multiple epigenome characteristics at once could both overcome the sparsity issue and derive important information about cell type-specific epigenome patterns at specific loci. However, we still lack methods for profiling multiple epigenome targets simultaneously in the same assay, in what might be considered "Multifactorial" profiling.

Motivated by this gap, and with the knowledge that CUT\&Tag profiles chromatin proteins in single cells at high signal-to-noise ratio ${ }^{16}$, we explored methods for physical association of a chromatin protein-targeting antibody with an identifying adapter barcode added during tagmentation that could be used to deconvolute epigenome targets directly in sequencing (Fig. 1a). Using antibodies against mutually exclusive H3K27me3 and PollIS5P in human K562 Chronic Myelogenous Leukemia cells as controls, we systematically tested a variety of protocol conditions for antibody-barcode association with the goal of optimizing both assay efficiency and fidelity of target identification. We 
first tested the pre-incubation of barcoded pA-Tn5 complexes with antibodies versus covalent conjugation of barcoded adapters to either primary or secondary antibodies to be loaded into pA-Tn5, and the combined incubation and tagmentation of all antibodies at the same time versus sequential tagmentation of targets one at a time. We found that both pre-incubation and combined tagmentation resulted in high levels of spurious crossenrichment between targets (Supplementary Fig. 1a), leading us to use adapterconjugated antibodies loaded into pA-Tn5 to tagment multiple targets in sequence. We next tested conjugating adapters directly to a primary antibody versus a secondary antibody, finding that the former resulted in superior target distinction (Fig. 1b-c, Supplementary Fig. 1a-b), but also variable data quality, likely owing to fewer pA-Tn5 complexes accumulating per target locus in the absence of a secondary antibody. To overcome this obstacle, we (1) Loaded pA-Tn5 onto $1^{\circ}$ antibody-conjugated i5 forward adapters, (2) Tagmented target chromatin in sequence, and (3) Added a secondary antibody followed by pA-Tn5 loaded exclusively with i7 reverse adapters and carried out a final tagmentation step (Fig. 1a). This resulted in libraries that were as robust as matched CUT\&Tag experiments, particularly for H3K27me3 (Supplementary Fig. 1c). We dubbed this combined approach Multiple Iargets Identified by Tagmentation (MulTI-Tag) (Fig. 1a).

In K562 cells and H1 human embryonic stem cells (hESCs), we simultaneously profiled three targets that represent distinct waypoints in the temporal trajectory of developmental gene expression: H3K27me3, enriched in developmentally regulated heterochromatin ${ }^{21,22}, \mathrm{H} 3 \mathrm{~K} 4 \mathrm{me} 2$, enriched at active enhancers and promoters ${ }^{23}$, and 
H3K36me3, co-transcriptionally catalyzed during transcription elongation ${ }^{24,25}$ (Fig. 1d-e). In comparison with control experiments in which each of the three targets was profiled individually, K562 and H1 MulTI-Tag both retain comparable efficiency of target-specific enrichment in peaks (Supplementary Fig. 2a-c). Moreover, both control and MulTI-Tag experiments exhibit characteristic patterns of enrichment for each mark, including H3K4me2 at promoters, H3K36me3 in gene bodies, and H3K27me3 across both (Fig. 1e, Supplementary Fig. 2d). Of note, in $\mathrm{H} 1$ hESCs only we observed overlap between H3K27me3 and H3K4me2 for both control and MulTI-Tag samples consistent with known "bivalent" chromatin ${ }^{26}$. These results show that MulTI-Tag retains high fidelity when scaled to multiple user-defined targets.

Given the successful adaptation of CUT\&Tag for single cell profiling ${ }^{16,27-29}$, we sought to use multifactorial profiling for single-cell molecular characterization (Fig. 2a). In an experiment profiling a mixture of human K562 cells and mouse NIH3T3 cells in single cell experiments, MulTI-Tag using either individual antibodies or multiple antibodies in combination showed similarly low cross-species collision rates $(10 \%, 12 \%, 13 \%$, and 17\%; for $\mathrm{H} 3 \mathrm{~K} 27 \mathrm{me} 3, \mathrm{H} 3 \mathrm{~K} 4 \mathrm{me} 2$, and $\mathrm{H} 3 \mathrm{~K} 36 \mathrm{me} 3$ alone; or all targets, respectively), indicating successful single cell isolation and profiling (Supplementary Fig. 3a). Moreover, pilot experiments in K562 cells profiled by MulTI-Tag yielded a comparable number of unique reads for each of the three targets as single cells profiled using only one antibody, indicating that MulTI-Tag is nearly additive under conditions in which amplification is saturating (Supplementary Fig. 3b). We therefore used MulTI-Tag to simultaneously profile H3K27me3, H3K4me2, and H3K36me3 in 348 K562 cells and 368 H1 cells (Fig. 
2b). We found that in the majority of peaks (59.1\% for H3K27me3; 95.7\% for H3K4me2; $94.5 \%$ for $\mathrm{H} 3 \mathrm{~K} 36 \mathrm{me} 3$ ), greater than $80 \%$ of fragments mapped within the peak were from the same target (Fig. 2c, Supplementary Fig. 4a-b), indicating that MulTI-Tag retains high specificity in single cells. We used Uniform Manifold Approximation and Projection $(\mathrm{UMAP})^{30,31}$ to project single cell data into low-dimensional space based on enriched features defined for each of the individual targets profiled and found that $\mathrm{H} 3 \mathrm{~K} 27 \mathrm{me} 3$ and H3K4me2 were able to distinguish cell types with $99.9 \%$ and $95.2 \%$ efficiency, respectively, confirming that MulTI-Tag generates data from multiple chromatin targets that are informative for cell type classification (Fig. 2d). Notably, H3K36me3 was insufficient to separate cell types (32.3\% efficiency), consistent with previous observations in single cell data and with the relative uniformity of $\mathrm{H} 3 \mathrm{~K} 36 \mathrm{me} 3$ enrichment across different cell types ${ }^{32}$. An analysis of the 248 most informative enriched features for cell type distinction based on their values in the Singular Value Decomposition (SVD) input to UMAP showed that $100 \%$ of them were $\mathrm{H} 3 \mathrm{~K} 27 \mathrm{me} 3$ features, consistent with its near-perfect distinction of highly dissimilar cell-type specific clusters (Supplementary Fig. 4c). Nevertheless, we were able to identify highly informative target-specific clusters for all three targets that showed cell type-specific patterns of enrichment (Supplementary Fig. 4d). These data show that MulTI-Tag is an effective method for profiling multiple informative chromatin targets in single cells.

Since MulTI-Tag uses barcoding to define fragments originating from specific targets, we can directly ascertain and quantify relative target abundances and instances of their cooccurrence at the same loci in single cells. By quantifying the number of unique reads 
occurring for each target, we found that H3K27me3 was highly abundant relative to H3K4me2 and H3K36me3 in single cells, composing a mean of $89.4 \%$ of unique reads in K562 cells and $80.0 \%$ of unique reads in $\mathrm{H} 1$ cells, as compared with $\mathrm{H} 3 \mathrm{~K} 4 \mathrm{me} 2$ contributing $5.1 \%$ and $8.8 \%$ and $\mathrm{H} 3 \mathrm{~K} 36 \mathrm{me} 3$ contributing $5.5 \%$ and $11.2 \%$ in $\mathrm{K} 562$ and H1 cells, respectively (Fig. 3a, Supplementary Fig. 5a). Although several variables could contribute to this difference, including antibody binding efficiency and signal-to-noise ratio, it is consistent with previously reported mass spectrometry quantification of H3K27me3- vs. H3K4me2-containing peptides ${ }^{33}$ and of single molecule imaging of H3K27me3- and H3K4me3-modified nucleosomes ${ }^{34}$ in hESCs, suggesting the relative balances of target-specific fragments reflect biologically meaningful quantifications.

To quantify co-occurrence of specific target combinations in single cells, we mapped fragments from any target onto genes in a window from $1 \mathrm{~kb}$ upstream of the TSS to the gene terminus and clustered cells based on a binarized, low dimensional representation of that signal. We then categorized genes in each cell based on the co-occurrence of target-specific fragments, and finally mapped those categories onto clustered cells (Fig. 3b, Supplementary Fig. 5b). As expected, due to the relative abundances of targetspecific fragments, the 363 most informative genes by all-target clustering were highly represented for $\mathrm{H} 3 \mathrm{~K} 27$ me3 enrichment in the absence of other targets (Fig. 3c). However, many notable genes exhibited unique patterns of target co-occurrence that correlated with cell type, including H3K27me3-H3K4me2 co-enriched at the CREB5 and ZNF423 genes in $\mathrm{H} 1$ cells in comparison with exclusive H3K27me3 enrichment in K562 cells (Fig. 3d). There are also notable instances of co-enrichment across genes in the same single 
cells, including cells with H3K4me2 and/or H3K36me3 enrichment in NR5A2 linked with H3K27me3 enrichment in HOXB3 in H1 cells, and vice-versa in K562 cells (Fig. 3d). These results show that multi-target intra-gene co-enrichment and cross-gene patterns can be directly observed in MulTI-Tag data.

We quantified the proportion of each class of single- or co-enrichment of targets in the same gene in single cells and found that although H3K27me3 in the absence of other targets is the most represented class, K562 cells have a significantly higher share of this class of genes than $\mathrm{H} 1$ cells, and a lower frequency of all other target combinations (Fig. 3e). This is consistent with the repeated observation that hESCs have a lower density of heterochromatin than many other cell types ${ }^{35,36}$. To quantify the degree of coordination between each pair of targets, we calculated Cramer's $V$ of association, a variant of Pearson's Chi-square test that is robust to variable effect sizes and is therefore ideal for MulTI-Tag data with highly disparate target-specific counts. We found that $\mathrm{H} 1$ cells had a higher degree of association between H3K27me3 and H3K4me2 than K562 cells, consistent with observed bivalency (Fig. 3f). Curiously, the same was true for association between H3K27me3 and H3K36me3, despite previous observations that H3K27me3 and H3K36me3 appear to be antagonistic in vitro and in vivo ${ }^{37,38}$ (Fig. 3F). Nevertheless, in bulk MulTI-Tag and in previously published ENCODE ChIP-seq data from $\mathrm{H} 1 \mathrm{hESCs}$, we were similarly able to detect co-occurrence of H3K27me3 at the 5' ends and H3K36me3 at the 3' ends of several genes, including many involved in metabolic and developmental signaling and others that appear to have multiple regulated promoter isoforms (Supplementary Fig. 6a-b), suggesting our observation of single-cell co-occurrence is 
unlikely to be a technical artifact. In contrast, we find that the association between H3K4me2 and H3K36me3 is low in both cell types, indicating that despite their independent associations with active transcription, the simultaneous enrichment of $\mathrm{H} 3 \mathrm{~K} 4 \mathrm{me} 2$ at the promoter and H3K36me3 in the body of a gene is not overrepresented relative to chance. Together, these results shed light on patterns of chromatin structure at single cell, single locus resolution.

MulTI-Tag establishes a rigorous baseline for unambiguously profiling multiple epigenome proteins with direct sequence tags, maintaining both exemplary assay efficiency and target-assignment fidelity relative to other similar approaches ${ }^{39,40}$. Singlereadout Multifactorial profiling holds a distinct advantage over Multimodal profiling, which often requires highly complicated integration of semi-compatible protocols and analysis methods. MulTI-Tag is theoretically scalable to any combination of user-defined targets in the same assay. Three targets profiled here, H3K27me3, H3K4me2, and H3K36me3, are typically enriched at distinct stages of the gene regulatory cycle that proceeds from developmental repression (H3K27me3) to enhancer and promoter activation (H3K4me2) to productive transcription elongation (H3K36me3). In the future we envision that integrating this temporal information across models of development and differentiation will aid in understanding the causative regulatory events that dictate how developmental transitions proceed at the single cell level prior to the downstream transcriptomic and proteomic outcomes. It will also be instructive to add targets to the same experiments that represent other waypoints in the cycle including RNA Polll pausing (PollIS5P) and elongation (PollIS2P), constitutive silencing (H3K9me3), and even direct transcription 
factor binding. Furthermore, our analysis of co-occurrence of different targets in the same genes elucidates chromatin structure at single-locus, single-cell resolution in a way that heretofore has been impossible. We anticipate further work to understand intra-locus interactions between different chromatin characteristics to bear on long-standing hypotheses regarding bivalency ${ }^{26}$ and hyperdynamic chromatin ${ }^{35}$.

Although MulTI-Tag represents a significant advance in chromatin profiling, opportunities for refinement exist. For instance, it is possible that PCR "jackpotting" bias may suppress the equitable amplification of some target combinations. Methods to mitigate targetspecific amplification bias could resolve this, though MulTI-Tag unique fragment depth appeared to be additive for the target combinations tested in this study. MulTI-Tag is also more complicated than CUT\&Tag, requiring additional steps and the use of adapterconjugated antibodies. Our emphasis on ensuring both that the efficiency of MulTI-Tag profiling was comparable to CUT\&Tag in terms of signal-to-noise, and that each individual target was faithfully assigned with minimal cross-contamination between antibodyassigned adapters, led us to generate antibody-adapter conjugates ${ }^{41}$, and to incubate and tagment with antibody-adapter-transposase complexes sequentially rather than simultaneously. By physically excluding the possibility of adapter or Tn5 monomer exchange in the protocol, MulTI-Tag safeguards against potential artifacts originating from adapter crossover, identifying any set of user-defined targets with high fidelity. We anticipate future reagent development and protocol improvements will enable methods in the style of MulTI-Tag to produce reliable multifactorial profiles while also minimizing the barriers to use for any lab seeking to obtain such data. 


\section{Methods}

\section{Cell culture and nuclei preparation}

Human female K562 Chronic Myleogenous Leukemia cells (ATCC) were authenticated for STR, sterility, human pathogenic virus testing, mycoplasma contamination, and viability at thaw. $\mathrm{H} 1$ (WA01) male human embryonic stem cells (hESCs) (WiCell) were authenticated for karyotype, STR, sterility, mycoplasma contamination, and viability at thaw. K562 cells were cultured in liquid suspension in IMDM (ATCC) with 10\% FBS added (Seradigm). $\mathrm{H} 1$ cells were cultured in Matrigel (Corning)-coated plates at $37^{\circ} \mathrm{C}$ and $5 \% \mathrm{CO}_{2}$ using mTeSR-1 Basal Medium (STEMCELL Technologies) exchanged every 24 hours. K562 cells were harvested by centrifugation for 3 mins at $1000 \times x g$, then resuspended in 1x Phosphate Buffered Saline (PBS). H1 cells were harvested with ReleasR (StemCell Technologies) using manufacturer's protocols. Lightly crosslinked nuclei were prepared from cells as described in steps 2-14 of the Bench Top CUT\&Tag protocol on protocols.io (https://dx.doi.org/10.17504/protocols.io.bcuhiwt6). Briefly, cells were pelleted 3 minutes at $600 x g$, resuspended in hypotonic NE1 buffer $(20$ mM HEPES$\mathrm{KOH} \mathrm{pH} \mathrm{7.9,} 10 \mathrm{mM} \mathrm{KCl}, 0.5 \mathrm{mM}$ spermidine, $10 \%$ Triton $\mathrm{X}-100,20 \%$ glycerol), and incubated on ice for 10 minutes. The mixture was pelleted 4 minutes at $1300 \mathrm{xg}$, resuspended in 1xPBS, and fixed with $0.1 \%$ Formaldehyde for 2 minutes before quenching with $60 \mathrm{mM}$ glycine. Nuclei were counted using the ViCell Automated Cell Counter (Beckman Coulter) and frozen at $-80^{\circ} \mathrm{C}$ in $10 \%$ DMSO for future use.

\section{Antibodies}


Antibodies used for CUT\&Tag or MulTI-Tag in this study were as follows: Rabbit AntiH3K27me3 (Cell Signaling Technologies CST9733S, Lot 16), Mouse anti-RNA PollIS5P (Abcam ab5408, Lot GR3264297-2), Mouse anti-H3K4me2 (Active Motif 39679, Lot 31718013), Mouse anti-H3K36me3 (Active Motif 61021, Lot 23819012), Guinea Pig antiRabbit (Antibodies Online ABIN101961), and Rabbit anti-Mouse (Abcam ab46450). For antibody-adapter conjugation, antibodies were ordered from manufacturers with the following specifications if not already available as such commercially: 1x PBS, no BSA, no Sodium Azide, no Glycerol. For secondary conjugate MulTI-Tag, secondary antibody conjugates from the TAM-ChIP Rabbit and Mouse kits (Active Motif) were used.

\section{CUT\&Tag}

CUT\&Tag was carried out as previously described $^{17}$ (https://dx.doi.org/10.17504/protocols.io.bcuhiwt6). Briefly, nuclei were thawed and bound to washed paramagnetic Concanavalin A (ConA) beads (Bangs Laboratories), then incubated with primary antibody at $4^{\circ} \mathrm{C}$ overnight in Wash Buffer $(10 \mathrm{mM}$ HEPES pH7.5, 150 mM NaCl, 0.5 mM spermidine, Roche Complete Protease Inhibitor Cocktail) with $2 \mathrm{mM}$ EDTA. Bound nuclei were washed and incubated with secondary antibody for 1 hour at room temperature (RT), then washed and incubated in Wash-300 Buffer (Wash Buffer with $300 \mathrm{mM} \mathrm{NaCl}$ ) with 1:200 loaded pA-Tn5 for 1 hour at RT. Nuclei were washed and tagmented in Wash-300 Buffer with $10 \mathrm{mM} \mathrm{MgCl}_{2}$ for 1 hour at $37^{\circ} \mathrm{C}$, then resuspended sequentially in $50 \mu \mathrm{L} 10 \mathrm{mM}$ TAPS and $5 \mu \mathrm{L} 10 \mathrm{mM}$ TAPS with $0.1 \%$ SDS, and incubated 1 hour at $58^{\circ} \mathrm{C}$. The resulting suspension was mixed well with $16 \mu \mathrm{L}$ of 0.9375\% Triton X-100, then primers and 2x NEBNext Master Mix (NEB) was added for 
direct amplification with the following conditions: 1) $58{ }^{\circ} \mathrm{C}$ for 5 minutes, 2) $72{ }^{\circ} \mathrm{C}$ for 5 minutes, 3) $98^{\circ} \mathrm{C}$ for 30 seconds, 4) $98{ }^{\circ} \mathrm{C} 10$ seconds, 5) $60{ }^{\circ} \mathrm{C}$ for 10 seconds, 6) Repeat steps 4-5 14 times, 7) $72{ }^{\circ} \mathrm{C}$ for 2 minutes, 8) Hold at $8{ }^{\circ} \mathrm{C}$. DNA from amplified product was purified using 1.1x ratio of HighPrep PCR Cleanup System (MagBio) and resuspended in $25 \mu \mathrm{L} 10 \mathrm{mM}$ Tris- $\mathrm{HCl}$ with $1 \mathrm{mM}$ EDTA, and concentration quantified using the TapeStation system (Agilent). For sequential and combined CUT\&Tag, rather than incubating the secondary antibody and pA-Tn5 separately, pA-Tn5 was preincubated with an equimolar amount of secondary antibody in $50 \mu \mathrm{LWash}-300$ buffer at $4^{\circ} \mathrm{C}$ overnight. For sequential, primary antibody incubation, secondary antibody-pA-Tn5 incubation, and tagmentation were carried out sequentially for each primary-secondarybarcoded pA-Tn5 combination, whereas for combined, all reagents were incubated simultaneously for their respective protocol steps (i.e. primary antibodies together, secondary antibody-pA-Tn5 complexes together), and tagmentation was carried out once for all targets.

\section{Conjugates for MulTI-Tag}

Antibody-adapter conjugates were generated by random amino-conjugation between 100 $\mu \mathrm{g}$ antibody purified in PBS in the absence of glycerol, BSA, and sodium azide, and 5' aminated, barcode-containing oligonucleotides (IDT) using Oligonucleotide Conjugation Kit (Abcam) according to manufacturer's protocols. Before conjugation, $200 \mu \mathrm{M}$ adapter oligos resuspended in 1xPBS were annealed to an equimolar amount of $200 \mu \mathrm{M}$ Tn5MErev (5'-[phos]CTGTCTCTTATACACATCT-3') in 1xPBS to yield $100 \mu \mathrm{M}$ annealed adapters. In all cases, primary antibodies were conjugated with an estimated 10:1 molar 
excess of adapter to conjugate. The sequences of adapters used are listed in Supplementary Table 1.

\section{Bulk MulTI-Tag protocol}

For each target to be profiled in MulTI-Tag, an antibody-i5 adapter conjugate was generated as described above, and $0.5 \mu \mathrm{g}$ conjugate was incubated with $1 \mu \mathrm{L}$ of $\sim 5 \mu \mathrm{M}$ pA-Tn5 and 16 pmol unconjugated, Tn5MErev-annealed i5 adapter of the same sequence in minimal volume for 30 minutes-1 hour at RT to generate conjugatecontaining i5 transposomes. In parallel, a separate aliquot of $1 \mu \mathrm{L} p A-\operatorname{Tn} 5$ was incubated with 32 pmol i7 adapter for 30 minutes-1 hour at RT to generate an i7 transposome. Conjugate i5 and i7 transposomes were used in MulTI-Tag experiments within 24 hours of assembly. After transposome assembly, 50000 nuclei were thawed and bound to washed ConA beads, then incubated with the first conjugate transposome resuspended in $50 \mu \mathrm{L}$ Wash-300 Buffer plus $2 \mathrm{mM}$ EDTA for 1 hour at RT or overnight at $4^{\circ} \mathrm{C}$. After incubation, the nuclei mix was washed 3 times with $200 \mu \mathrm{L}$ Wash-300 Buffer, then tagmented in $50 \mu \mathrm{L}$ Wash-300 Buffer with $10 \mathrm{mM} \mathrm{MgCl} 2$ for 1 hour at $37^{\circ} \mathrm{C}$. After tagmentation, buffer was removed and replaced with $200 \mu \mathrm{L}$ Wash-300 with 5 mM EDTA and incubated 5 minutes with rotation. The conjugate incubation and tagmentation protocol was then repeated for the remainder of conjugates to be used, up to the point of incubation with the final conjugate. The optimal order of conjugate tagmentation was ascertained empirically by observing the optimal balance of reads between targets, and in this study were tagmented in the following order: PollIS5P-H3K27me3; or H3K4me2H3K36me3-H3K27me3. After incubation, the supernatant was cleared and secondary 
antibodies corresponding to the species in which the primary antibody conjugates were raised were added in $100 \mu \mathrm{L}$ Wash Buffer and incubated for 1 hour at RT. The nuclei were then washed twice with $200 \mu \mathrm{L}$ Wash Buffer and the i7 transposome was added in $100 \mu \mathrm{L}$ Wash-300 Buffer, and incubated 1 hour at RT. After three washes with $200 \mu \mathrm{L}$ Wash-300 Buffer, the final tagmentation is carried out by adding $50 \mu \mathrm{L}$ Wash-300 Buffer with $10 \mathrm{mM} \mathrm{MgCl}_{2}$ and incubating 1 hour at $37^{\circ} \mathrm{C}$. After tagmentation, the nuclei are resuspended in $10 \mathrm{mM}$ TAPS, denatured in TAPS-SDS, neutralized in Triton X-100, amplified and libraries purified as described above. All nuclei transfers were carried out in low-bind $0.6 \mathrm{~mL}$ tubes (Axygen). For combined MulTI-Tag, all antibody conjugate incubation and tagmentation steps were carried out simultaneously.

\section{Single cell MulTI-Tag}

Single cell MulTI-Tag was carried out as described in Bulk MulTI-Tag protocol up to the completion of the final tagmentation step, with the following modifications: $250 \mu \mathrm{L}$ paramagnetic Streptavidin T1 Dynabeads (Sigma-Aldrich) were washed 3 times with 1 $\mathrm{mL} 1 \times$ PBS and resuspended in $1 \mathrm{~mL} 1 \times$ PBS with $0.01 \%$ Tween-20, $240 \mu \mathrm{L}$ of BiotinWheat Germ Agglutinin (WGA) (Vector Labs) combined with $260 \mu \mathrm{L}$ 1x PBS was incubated with dynabeads for 30 minutes and resuspended in $1 \mathrm{~mL} 1 \mathrm{x}$ PBS with $0.01 \%$ Tween-20 to generate WGA beads, and $100 \mu \mathrm{L}$ of washed beads were pre-bound with 6 million nuclei. For each experiment, $15 \mu \mathrm{g} \mathrm{H} 3 \mathrm{~K} 4 \mathrm{me} 2$ and H3K36me3 conjugate and 7.5 $\mu \mathrm{g} \mathrm{H} 3 \mathrm{~K} 27 \mathrm{me} 3$ conjugate were used, loaded into transposomes at the ratios described above. All incubations were carried out in $200 \mu \mathrm{L}$, and washes in $400 \mu \mathrm{L}$. After final conjugate and secondary antibody incubation, nuclei were distributed equally across i7 
transposomes containing 96 uniquely barcoded adapters (Supplementary Table 1). After the final tagmentation step, nuclei were reaggregated into a single tube, washed twice in $100 \mu \mathrm{L} 10 \mathrm{mM}$ TAPS, and transferred to a cold block chilled to $0^{\circ} \mathrm{C}$ on ice. Supernatant was removed and nuclei were incubated in ice cold DNase reaction mix (10 $\mu \mathrm{L}$ RQ1 DNase, (Promega), $10 \mu \mathrm{L}$ 10x DNase buffer, $80 \mu \mathrm{L} d \mathrm{dH}_{2} \mathrm{O}$ ) for 10 minutes in cold block. The reaction was stopped by adding $100 \mu \mathrm{L}$ ice cold RQ1 DNase Stop Buffer. Nuclei were immediately washed once in $100 \mu \mathrm{L} 10 \mathrm{mM}$ TAPS and then resuspended in $650 \mu \mathrm{L}$ TAPS. Two 20-micron cell strainers (Fisher Scientific) were affixed to fresh $1.5 \mathrm{~mL}$ low bind tubes, and $325 \mu \mathrm{L}$ nuclei mix was added to the top of each. Tubes were spun 10 minutes at $300 \mathrm{xg}$ to force nuclei through strainer, flowthrough was combined, and resuspended in $640 \mu \mathrm{L} 10 \mathrm{mM}$ TAPS. To the final nuclei mix, $16 \mu \mathrm{L} 100 x$ DAPI and $8 \mu \mathrm{L}$ ICELL8 Second Diluent (Takara) were added and incubated 10 minutes at RT. The entire nuclei mix was dispensed into an ICELL8 microfluidic chip according to manufacturer's protocols, and SDS denaturation, Triton $\mathrm{X}-100$ neutralization, and amplification were carried out in microwells as described previously ${ }^{32}$. After amplification, microwell contents were reaggregated and libraries were purified with two rounds of cleanup with 1.3x HighPrep beads and resuspended in $20 \mu \mathrm{L} 10 \mathrm{mM}$ Tris- $\mathrm{HCl}$ with $1 \mathrm{mM}$ EDTA.

\section{Sequencing and data preprocessing}

Libraries were sequenced on an Illumina HiSeq instrument with paired end 25x25 reads.

Sequencing data were aligned to the UCSC hg19 genome build using bowtie ${ }^{42}$, version 2.2.5, with parameters --end-to-end--very-sensitive--no-mixed--no-discordant -qphred33 -I 10 -X 700. Mapped reads were converted to paired-end BED files containing 
coordinates for the termini of each read pair, and then converted to bedgraph files using bedtools genomecov with parameter $-\mathrm{bg}^{43}$. For single cell experiments, mapped reads were converted to paired-end CellRanger-style bed files, in which the fourth column denotes cell barcode combination, and the fifth column denotes the number of fragment duplicates. Raw read counts and alignment rates for all sequencing datasets presented in this study are listed in Supplementary Table 2.

\section{Data Availability Statement}

All primary sequence data and interpreted track files for sequence data generated in this study have been deposited at Gene Expression Omnibus (GEO): GSE179756. Publicly available CUT\&Tag and ChIP-seq data analyzed in this study are found at GSE124557, and at the ENCODE Portal at the UCSC Genome Browser (http://hgdownload.soe.ucsc.edu/goldenPath/hg19/encodeDCC/wgEncodeBroadHistone Ц), respectively.

\section{Data Analysis}

Code necessary for the analyses performed in this study are available on Github (https://github.com/mpmeers/MeersEtAl MulTI-Tag). Single cell MulTI-Tag preprocessing, feature selection, dimensionality reduction and UMAP projection were carried out as follows: for each target, a unique fragments per cell cutoff ( 500 for H3K27me3, 200 for H3K4me2, 200 for H3K36me3) was selected based on knee plot analysis, and cells were retained only if they met unique read count criteria for all three targets. For bulk MuLTI-Tag, peaks were called using SEACR v1.4 $4^{44}$ with the following settings: $-\mathrm{n}$ norm, 
-m stringent, -e 0.1 (https://github.com/FredHutch/SEACR). For single cell MulTI-Tag, peaks were called from aggregate profiles from unique read count-filtered cells using SEACR v1.4 with the following settings: -n norm, -m stringent, -e 5. Peak calls presented in this study are listed in Supplementary Table 3. Cell-specific unique reads were intersected with peaks using Bedtools ${ }^{43}$ to generate bed files in which each line contained a unique peak-cell-read count instance. In R (https://www.r-project.org), these bed files were cast into peak (rows) by cell (columns) matrices, which were filtered for the top $40 \%$ of peaks by aggregate read counts, scaled by term frequency-inverse document frequency (TF-IDF), and log-transformed. For the gene-centric analysis presented in Figure 3, fragments were mapped to genes in a window extending from $1 \mathrm{~kb}$ upstream of the fathest distal annotated TSS to the annotated TES, and matrices were binarized before filtering and TF-IDF processing. Transformed matrices were subjected to Singular Value Decomposition (SVD), and SVD dimensions for which the values in the diagonal matrix ( $\$ d$ as output from the "svd" command in $R$ ) were greater than $0.2 \%$ of the sum of all diagonal values were used as input to the "umap" command from the umap library in R. Variable features for heatmap plotting were defined as those in the $99^{\text {th }}$ percentile of absolute value in the first or second component of the SVD transformation, or those in the $99^{\text {th }}$ percentile of target-specific features. For genic co-occurrence analysis, the statistical significance of cell-specific, target-specific fragment accumulation in genes was verified by calculating the probability of $X$ fragment-gene overlaps in cell $i$ based on a poisson distribution with a mean $\mu_{i}$ defined by the cell-specific likelihood of a fragment overlap with any base pair in the hg19 reference genome:

$$
p=\text { Poisson }\left(X \geq x, \mu_{i}\right) ; \text { where } x=\frac{r * L_{i}}{L_{\text {gene }}} \text { and } \mu_{i}=\frac{L_{i} * f_{i}}{L_{\text {genome }}}
$$


Where $L_{i}=$ median fragment size in cell $i, f_{i}=$ number of fragments mapping in cell $i, L_{g e n e}$ $=$ length of the gene being tested, and $L_{\text {genome }}=$ length of the reference genome. All genefragment overlaps considered in this study were determined to be statistically significant at a $p<0.01$ cutoff after Benjamini-Hochberg multiple testing correction. P-values comparing target combination proportions in single cells were calculated using two-sided t-tests. All underlying statistics associated with statistical comparisons presented in this study are listed in Supplementary Table 4. Genome browser screenshots were obtained from Integrative Genomics Viewer (IGV) ${ }^{45}$. CUT\&Tag/MulTI-Tag enrichment heatmaps and average plots were generated in DeepTools ${ }^{46}$. UMAPs, violin plots, scatter plots and knee plots were generated using ggplot2 (https://ggplot2.tidyverse.org). Single cell enrichment heatmap displays and hierarchical clustering were generated using the "heatmap" utility and base R graphics. Ternary plots were generated using the ggtern library (http://www.ggtern.com/).

\section{Acknowledgments}

We thank Trizia Llagas, Terri Bryson, and Christine Codomo for technical support and Jorja Henikoff and Matthew Fitzgibbon for bioinformatics support for the experiments described in this manuscript. We also thank Kami Ahmad and members of the Henikoff Lab for manuscript critiques, Manu Setty for crucial advice on statistical validation, and Hatice Kaya-Okur for early inspiration and continuing advice throughout the development of this study. This work was supported by the Howard Hughes Medical Institute, an NIH Postdoctoral Fellowship to MPM (F32 GM129954) and an NIH R01 to SH (R01 HG010492). 


\section{Author Contributions}

MPM conceived the study, carried out the experiments, analyzed the data, and wrote the manuscript. DHJ developed and advised on methods for single cell isolation on the Takara ICELL8 microfluidic platform. SH provided funding, guidance on experiments, and critical and editing support for the manuscript.

\section{Competing Interests Statement}

The authors declare no competing interests.

\section{References}

$1 \quad$ Klein, A. M. et al. Droplet barcoding for single-cell transcriptomics applied to embryonic stem cells. Cell 161, 1187-1201, doi:10.1016/j.cell.2015.04.044 (2015).

2 Macosko, E. Z. et al. Highly Parallel Genome-wide Expression Profiling of Individual Cells Using Nanoliter Droplets. Cell 161, 1202-1214, doi:10.1016/j.cell.2015.05.002 (2015).

3 Cao, J. et al. Comprehensive single-cell transcriptional profiling of a multicellular organism. Science 357, 661-667, doi:10.1126/science.aam8940 (2017).

4 Bandura, D. R. et al. Mass cytometry: technique for real time single cell multitarget immunoassay based on inductively coupled plasma time-of-flight mass spectrometry. Anal Chem 81, 6813-6822, doi:10.1021/ac901049w (2009). 
5 Bendall, S. C. et al. Single-cell mass cytometry of differential immune and drug responses across a human hematopoietic continuum. Science 332, 687-696, doi:10.1126/science.1198704 (2011).

6 Shahi, P., Kim, S. C., Haliburton, J. R., Gartner, Z. J. \& Abate, A. R. Abseq: Ultrahigh-throughput single cell protein profiling with droplet microfluidic barcoding. Sci Rep 7, 44447, doi:10.1038/srep44447 (2017).

7 Guo, H. et al. Single-cell methylome landscapes of mouse embryonic stem cells and early embryos analyzed using reduced representation bisulfite sequencing. Genome Res 23, 2126-2135, doi:10.1101/gr.161679.113 (2013).

8 Smallwood, S. A. et al. Single-cell genome-wide bisulfite sequencing for assessing epigenetic heterogeneity. Nat Methods 11, 817-820, doi:10.1038/nmeth.3035 (2014).

9 Buenrostro, J. D. et al. Single-cell chromatin accessibility reveals principles of regulatory variation. Nature 523, 486-490, doi:10.1038/nature14590 (2015).

10 Cusanovich, D. A. et al. Multiplex single cell profiling of chromatin accessibility by combinatorial cellular indexing. Science $\quad 348, \quad 910-914$, doi:10.1126/science.aab1601 (2015).

11 Cao, J. et al. Joint profiling of chromatin accessibility and gene expression in thousands of single cells. Science 361, 1380-1385, doi:10.1126/science.aau0730 (2018).

12 Stoeckius, M. et al. Simultaneous epitope and transcriptome measurement in single cells. Nat Methods 14, 865-868, doi:10.1038/nmeth.4380 (2017). 
$13 \mathrm{Hu}, \mathrm{Y}$. et al. Simultaneous profiling of transcriptome and DNA methylome from a single cell. Genome Biol 17, 88, doi:10.1186/s13059-016-0950-z (2016).

14 Clark, S. J. et al. scNMT-seq enables joint profiling of chromatin accessibility DNA methylation and transcription in single cells. Nat Commun 9, 781, doi:10.1038/s41467-018-03149-4 (2018).

15 Swanson, E. et al. Simultaneous trimodal single-cell measurement of transcripts, epitopes, and chromatin accessibility using TEA-seq. Elife 10, doi:10.7554/eLife.63632 (2021).

16 Kaya-Okur, H. S. et al. CUT\&Tag for efficient epigenomic profiling of small samples and single cells. Nat Commun 10, 1930, doi:10.1038/s41467-019-09982-5 (2019).

17 Kaya-Okur, H. S., Janssens, D. H., Henikoff, J. G., Ahmad, K. \& Henikoff, S. Efficient low-cost chromatin profiling with CUT\&Tag. Nat Protoc 15, 3264-3283, doi:10.1038/s41596-020-0373-x (2020).

18 Ernst, J. \& Kellis, M. Discovery and characterization of chromatin states for systematic annotation of the human genome. Nat Biotechnol 28, 817-825, doi:10.1038/nbt.1662 (2010).

19 Consortium, E. P. An integrated encyclopedia of DNA elements in the human genome. Nature 489, 57-74, doi:10.1038/nature11247 (2012).

20 Spitz, F. \& Furlong, E. E. Transcription factors: from enhancer binding to developmental control. Nat Rev Genet 13, 613-626, doi:10.1038/nrg3207 (2012).

21 Cao, R. et al. Role of histone H3 lysine 27 methylation in Polycomb-group silencing. Science 298, 1039-1043, doi:10.1126/science.1076997 (2002). 
22 Boyer, L. A. et al. Polycomb complexes repress developmental regulators in murine embryonic stem cells. Nature 441, 349-353, doi:10.1038/nature04733 (2006).

23 Barski, A. et al. High-resolution profiling of histone methylations in the human genome. Cell 129, 823-837, doi:10.1016/j.cell.2007.05.009 (2007).

24 Bannister, A. J. et al. Spatial distribution of di- and tri-methyl lysine 36 of histone H3 at active genes. J Biol Chem 280, 17732-17736, doi:10.1074/jbc.M500796200 (2005).

25 Wagner, E. J. \& Carpenter, P. B. Understanding the language of Lys36 methylation at histone H3. Nat Rev Mol Cell Biol 13, 115-126, doi:10.1038/nrm3274 (2012).

26 Bernstein, B. E. et al. A bivalent chromatin structure marks key developmental genes in embryonic stem cells. Cell 125, 315-326, doi:10.1016/j.cell.2006.02.041 (2006).

27 Bartosovic, M., Kabbe, M. \& Castelo-Branco, G. Single-cell CUT\&Tag profiles histone modifications and transcription factors in complex tissues. Nat Biotechnol, doi:10.1038/s41587-021-00869-9 (2021).

28 Wu, S. J. et al. Single-cell CUT\&Tag analysis of chromatin modifications in differentiation and tumor progression. Nat Biotechnol, doi:10.1038/s41587-02100865-z (2021).

29 Zhu, C. et al. Joint profiling of histone modifications and transcriptome in single cells from mouse brain. Nat Methods 18, 283-292, doi:10.1038/s41592-02101060-3 (2021). 
30 Mclnnes, L., Healy, J. \& Melville, J. UMAP: Uniform Manifold Approximation and Projection for Dimension Reduction. arXiv:1802.03426 (2018). <https://ui.adsabs.harvard.edu/abs/2018arXiv180203426M>.

31 Becht, E. et al. Dimensionality reduction for visualizing single-cell data using UMAP. Nat Biotechnol, doi:10.1038/nbt.4314 (2018).

32 Janssens, D. H. et al. Automated CUT\&amp;Tag profiling of chromatin heterogeneity in mixed-lineage leukemia. bioRxiv, 2020.2010.2006.328948, doi:10.1101/2020.10.06.328948 (2021).

33 Leroy, G. et al. A quantitative atlas of histone modification signatures from human cancer cells. Epigenetics Chromatin 6, 20, doi:10.1186/1756-8935-6-20 (2013).

34 Shema, E. et al. Single-molecule decoding of combinatorially modified nucleosomes. Science 352, 717-721, doi:10.1126/science.aad7701 (2016).

35 Meshorer, E. et al. Hyperdynamic plasticity of chromatin proteins in pluripotent embryonic stem cells. Dev Cell 10, 105-116, doi:10.1016/j.devcel.2005.10.017 (2006).

36 Hawkins, R. D. et al. Distinct epigenomic landscapes of pluripotent and lineagecommitted human cells. Cell Stem Cell 6, 479-491, doi:10.1016/j.stem.2010.03.018 (2010).

37 Yuan, W. et al. H3K36 methylation antagonizes PRC2-mediated H3K27 methylation. J Biol Chem 286, 7983-7989, doi:10.1074/jbc.M110.194027 (2011).

38 Lu, C. et al. Histone H3K36 mutations promote sarcomagenesis through altered histone methylation landscape. Science 352, 844-849, doi:10.1126/science.aac7272 (2016). 
39 Handa, T. et al. Chromatin integration labeling for mapping DNA-binding proteins and modifications with low input. Nat Protoc 15, 3334-3360, doi:10.1038/s41596020-0375-8 (2020).

40 Gopalan, S., Wang, Y., Harper, N. W., Garber, M. \& Fazzio, T. G. Simultaneous profiling of multiple chromatin proteins in the same cells. bioRxiv, 2021.2004.2027.441642, doi:10.1101/2021.04.27.441642 (2021).

41 Harada, A. et al. A chromatin integration labelling method enables epigenomic profiling with lower input. Nat Cell Biol 21, 287-296, doi:10.1038/s41556-018-02483 (2019).

42 Langmead, B. \& Salzberg, S. L. Fast gapped-read alignment with Bowtie 2. Nat Methods 9, 357-359, doi:10.1038/nmeth.1923 (2012).

43 Quinlan, A. R. \& Hall, I. M. BEDTools: a flexible suite of utilities for comparing genomic features. Bioinformatics 26, 841-842, doi:10.1093/bioinformatics/btq033 (2010).

44 Meers, M. P., Tenenbaum, D. \& Henikoff, S. Peak calling by Sparse Enrichment Analysis for CUT\&RUN chromatin profiling. Epigenetics Chromatin 12, 42, doi:10.1186/s13072-019-0287-4 (2019).

45 Robinson, J. T. et al. Integrative genomics viewer. Nat Biotechnol 29, 24-26, doi:10.1038/nbt.1754 (2011).

46 Ramirez, F. et al. deepTools2: a next generation web server for deep-sequencing data analysis. Nucleic Acids Res 44, W160-165, doi:10.1093/nar/gkw257 (2016).

\section{Figure Legends}


Figure 1: MulTI-Tag directly identifies user-defined chromatin targets in the same cells.

a) Schematic describing the MulTI-Tag methodology: 1) Antibody-oligonucleotide conjugates are used to physically associate forward-adapter barcodes with targets, and are loaded directly into pA-Tn5 transposomes for sequential binding and tagmentation; 2) pA-Tn5 loaded exclusively with reverse adapters are used for a secondary CUT\&Tag step to efficiently introduce the reverse adapter to conjugate-bound loci; 3) Target-specific profiles are distinguished by barcode identity in sequencing. b) Genome browser screenshot showing individual CUT\&Tag profiles for H3K27me3 (first row) and RNA PollIS5P (second) in comparison with MulTI-Tag profiles for the same targets probed individually in different cells (third and fourth rows) or sequentially in the same cells (fifth and sixth). c) Heatmaps describing the enrichment of H3K27me3 (red) or RNA PollIS5P (blue) signal from sequential MulTI-Tag profiles at CUT\&Tag-defined H3K27me3 peaks (left) or RNA PollIS5P peaks (right). d) Genome browser screenshot showing H3K27me3 (red), H3K4me2 (purple), and H3K36me3 (teal) MulTI-Tag signal from experiments in $\mathrm{H} 1$ hESCs using an individual antibody (rows 1, 3, and 5) or all three antibodies in sequence (rows 2, 4, and 6). e) Top: Schematic showing characteristic enrichment of H3K27me3 (red), H3K4me2 (purple), and H3K36me3 (teal) across genes. Bottom: Normalized CUT\&Tag (light colors) and MulTI-Tag (dark colors) enrichment of H3K27me3, H3K4me2, and H3K36me3 across genes in H1 hESCs.

Figure 2: MulTI-Tag in single cells. a) Schematic describing single cell MulTI-Tag experiments. H1 hESCs (red) and K562 cells (green) were profiled separately in bulk, then individual cells were dispensed into nanowells on a Takara ICELL8 microfluidic 
device for single cell barcoding via amplification. b) Genome browser screenshot showing single cell MulTI-Tag data from K562 (top) and H1 (bottom) cells. Target-specific enrichment is shown in aggregate in first three columns, and cell-specific fragments for the top 50 cells by unique fragments per cell are shown below. c) Ternary plot describing the percentage of normalized fragments originating from H3K27me3 (left axis), H3K4me2 (right axis), or H3K36me3 (bottom axis) in each peak (open circles) called from aggregate K562 cell MulTI-Tag. Peak identity is denoted by circle color, and total normalized fragments is denoted by circle size. d) UMAP plots for single cell MulTI-Tag data in H1 and K562 cells. Projections based on H3K27me3 (left), H3K4me2 (center left), H3K36me3 (center right), or all features combined (right) are shown.

Figure 3: Coordinate multifactorial analysis in the same cells using MulTI-Tag. a) Violin plots describing the distribution of the proportions of MulTI-Tag H3K27me3 (red), H3K4me2 (purple), or H3K36me3 (teal) unique reads out of total unique reads in individual cells, with points representing each single cell value. Lines connect points that represent the same individual cell. b) Schematic describing coordinated multifactorial analysis strategy for MulTI-Tag. Genes in individual cells are analyzed for the enrichment of all MulTI-tag targets, and gene-cell target combinations are mapped onto a matrix for clustering and futher analysis. c) Heatmap describing co-occurrence of MulTI-tag targets in 363 highly variable genes in each of 716 single cells. The heatmap is hierarchically clustered on the column side by normalized all-factor enrichment, and on the row side by displayed co-occurrence values. Heatmap color code describes co-occurrence representations; color coding above columns indicates cell identity. d) Heatmap column- 
clustered as in c) describing co-occurrence in 6 genes of interest. e) Violin plots describing the distributions of proportions of each co-occurrence state as described below the plot in individual H1 (red) or K562 (green) cells, with points denoting individual cell values. The last six co-occurrence states are rescaled and inset at top right; $p$-values derived from two-sided student's t-test comparing distributions between cell types are listed above violins (not corrected for multiple hypothesis testing). f) Violin plots describing calculated Cramer's $\mathrm{V}$ of Association between target combinations listed at bottom in individual $\mathrm{H} 1$ (red) or K562 (green) cells, with points denoting individual cell values. 


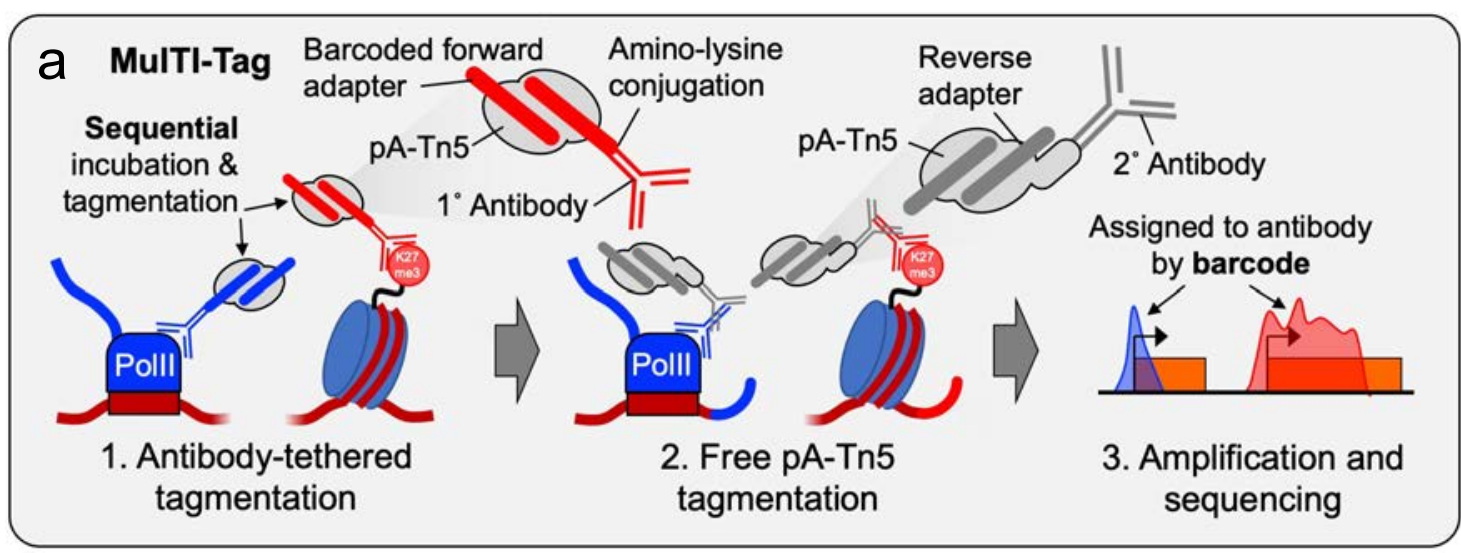

Figure 1

\section{b $\square$ H3K27me3 $\square$ PollIS5P}

Antibody

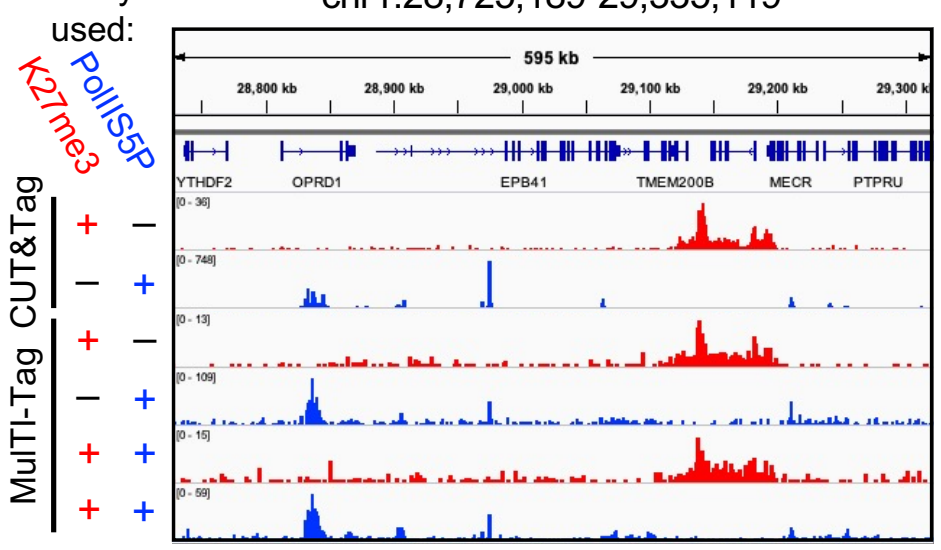

\section{d}

Antibody used:

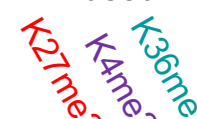

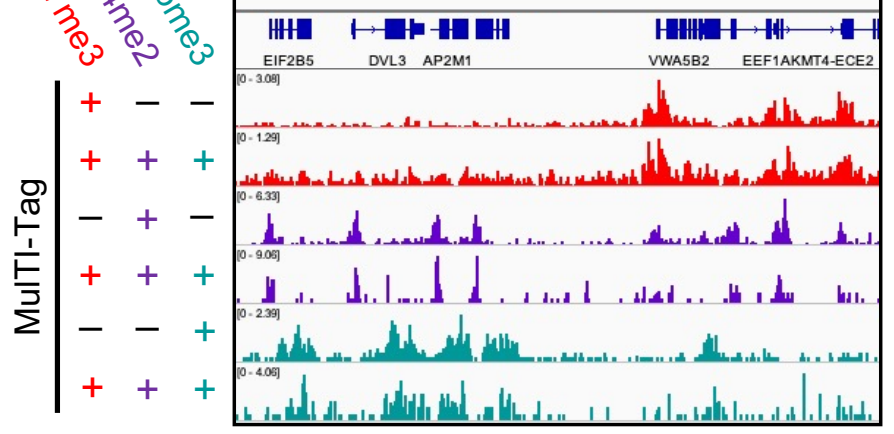

C H3K27me3 peaks PollIS5P peaks
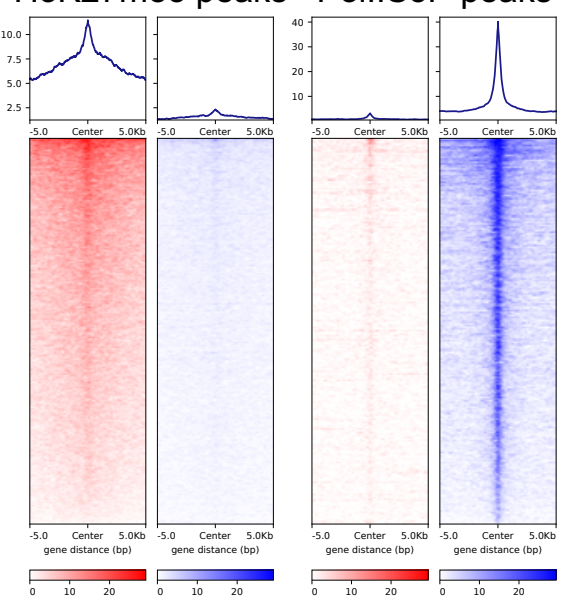

MulTI-Tag

H3K27me3

PollIS5P

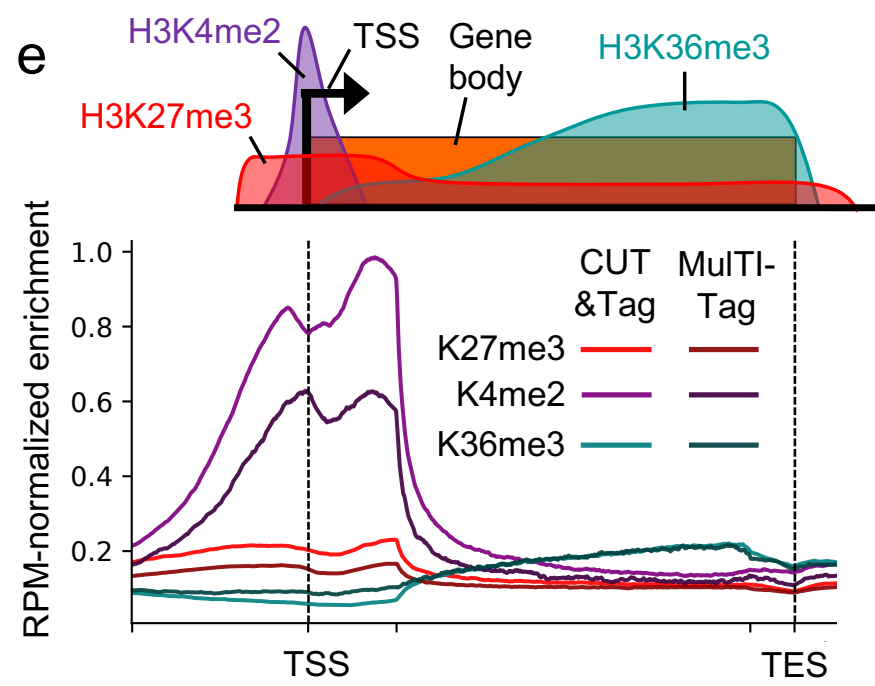


a

Figure 2

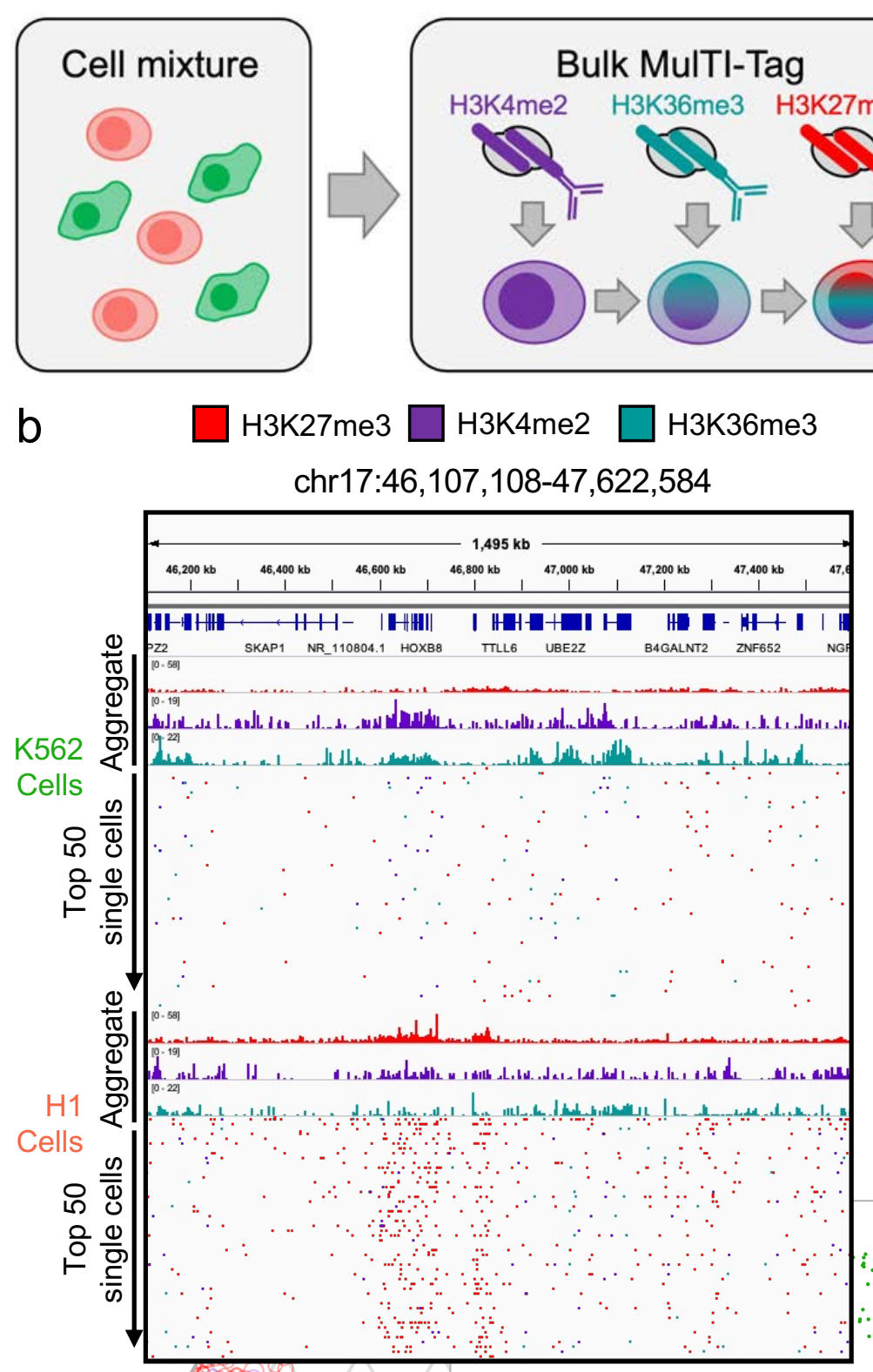

d

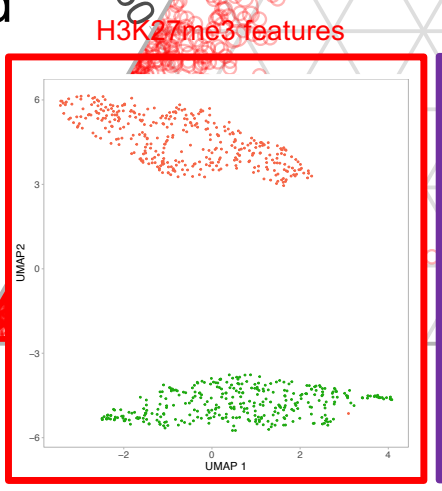

H3K4me2 features
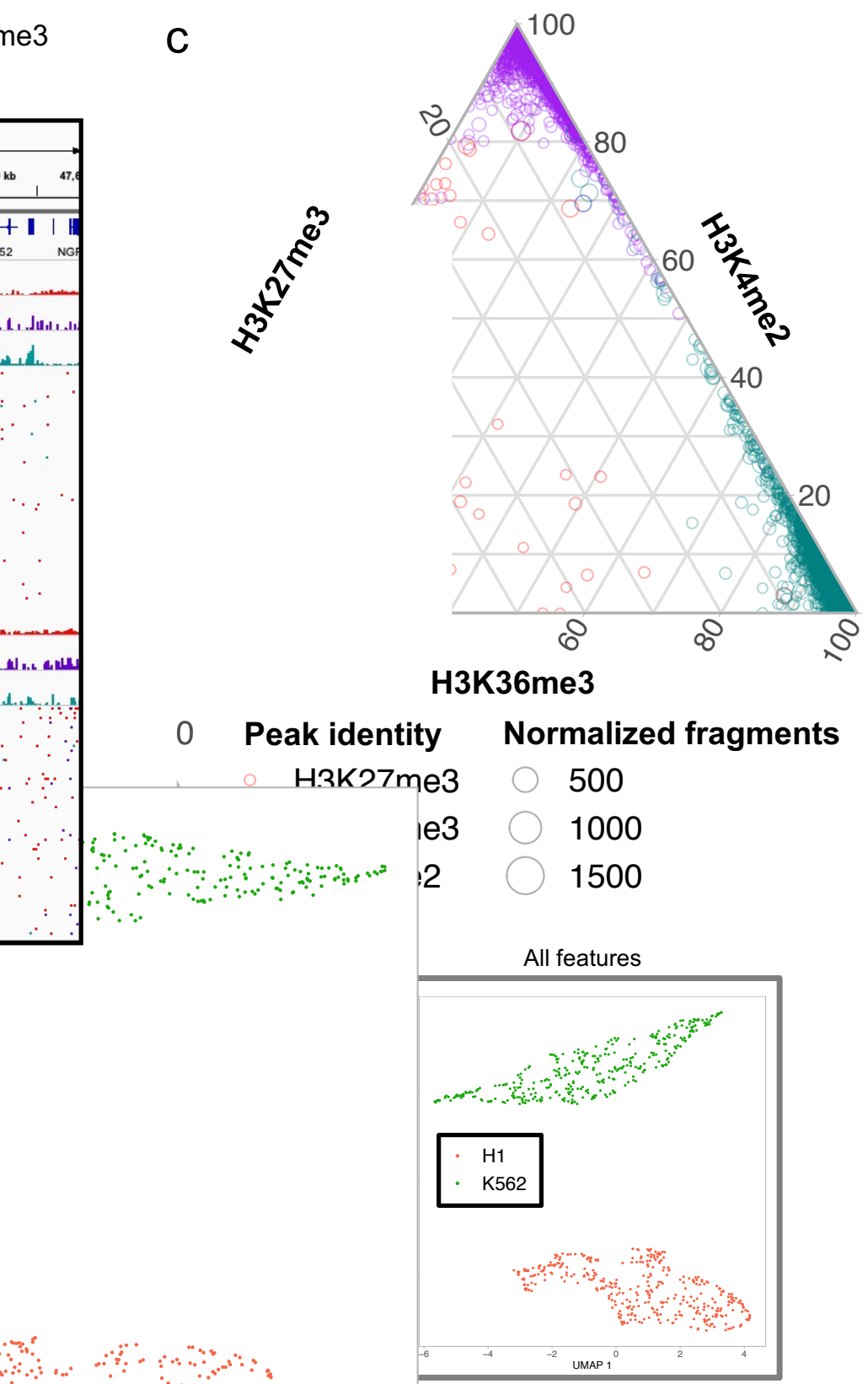

\section{Peak identity Normalized fragments}

H3K27me3 $\bigcirc 500$

H3K36me3 1000

H3K4me2 1500

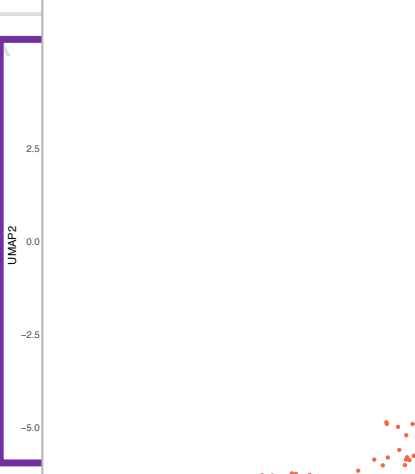

UMAP 1 
a

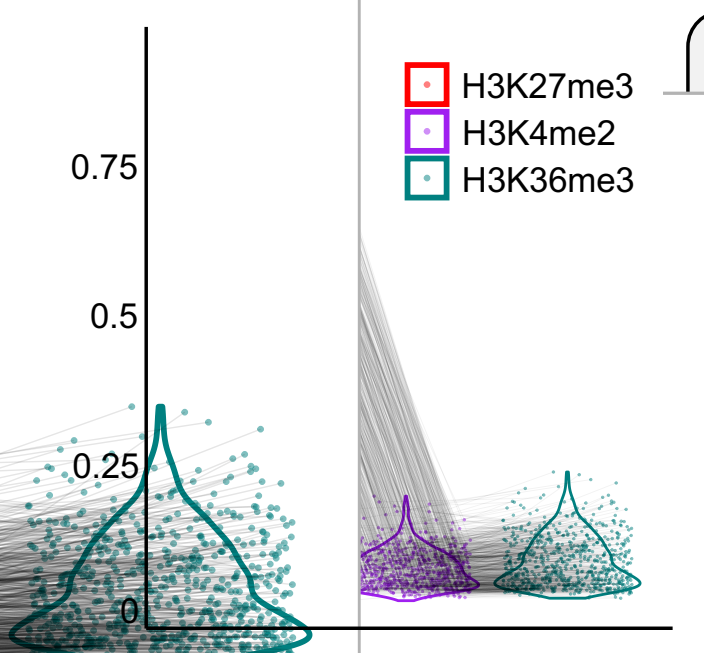

C

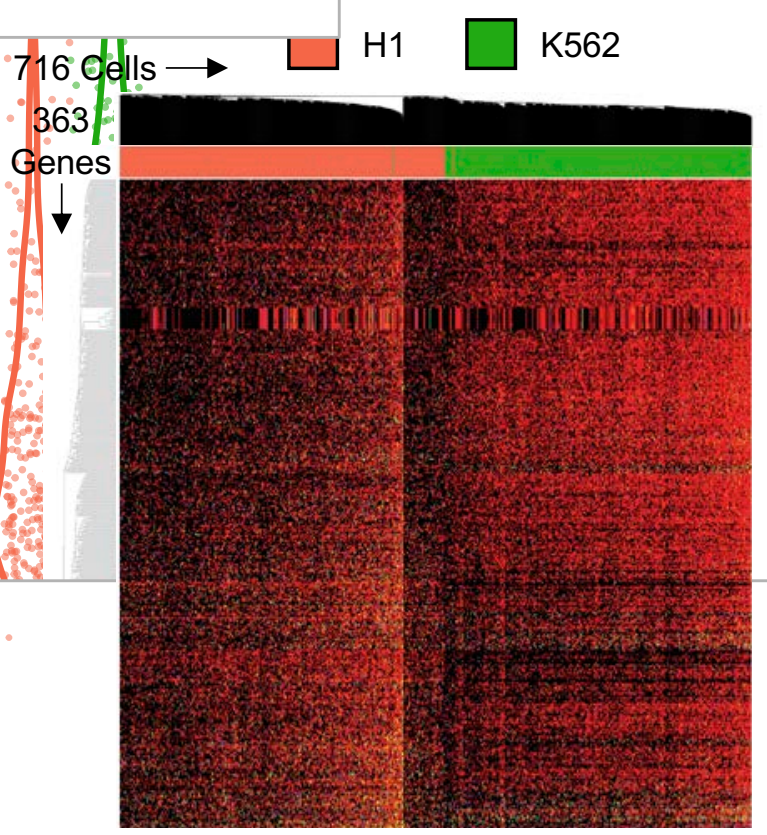

Heatmap

color:

H3K27me3: -+--+-++

H3K4me2: --+-++-+

H3K36me3: --+++++

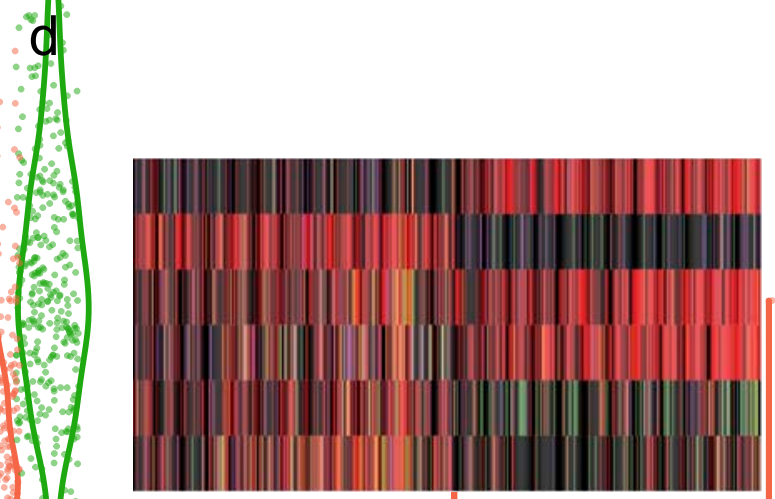

Figure 3

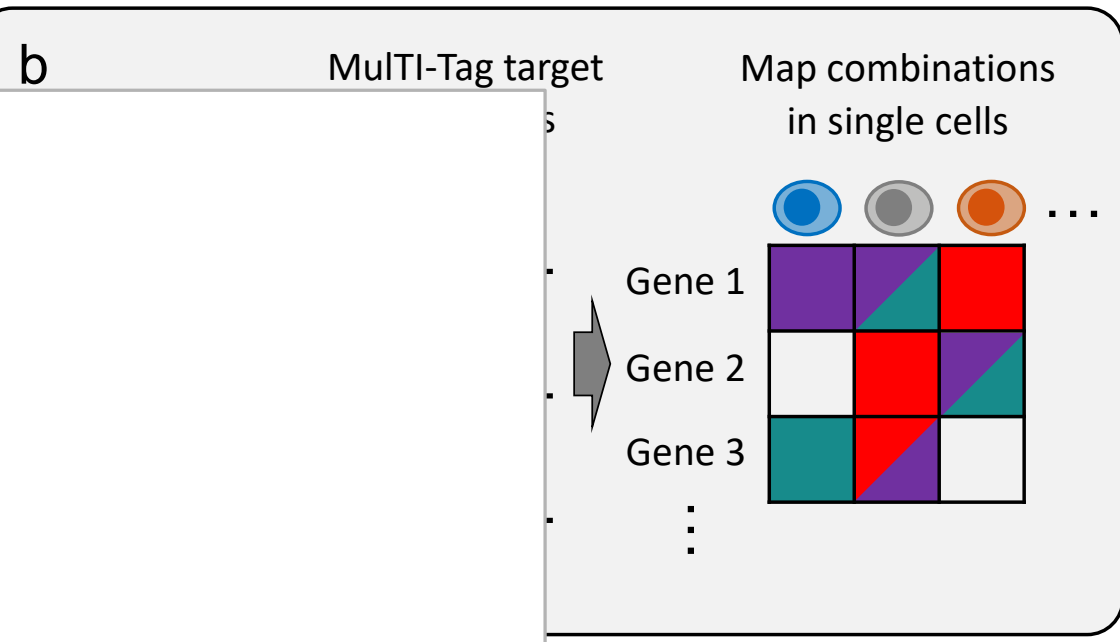

e $4.62 \mathrm{E}-12$

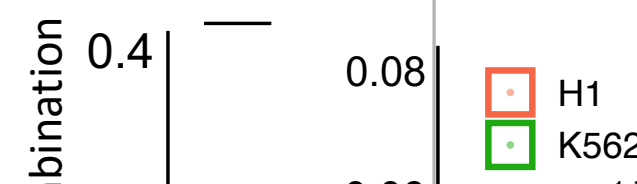

6.54E-8 

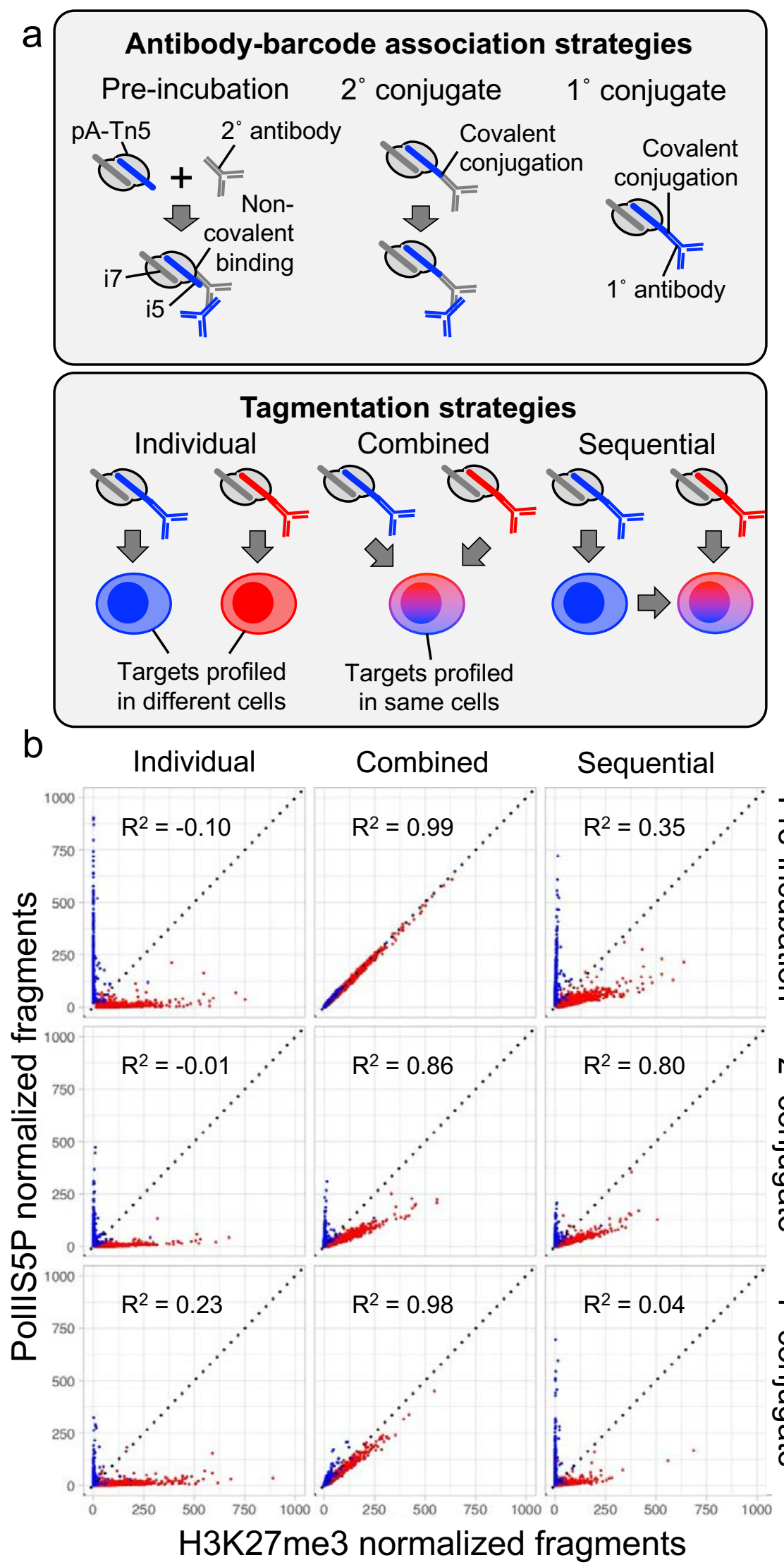

Supplementary Figure 1

C

$\square$ H3K27me3 $\square$ PollIS5P chr1: 29,111,801-

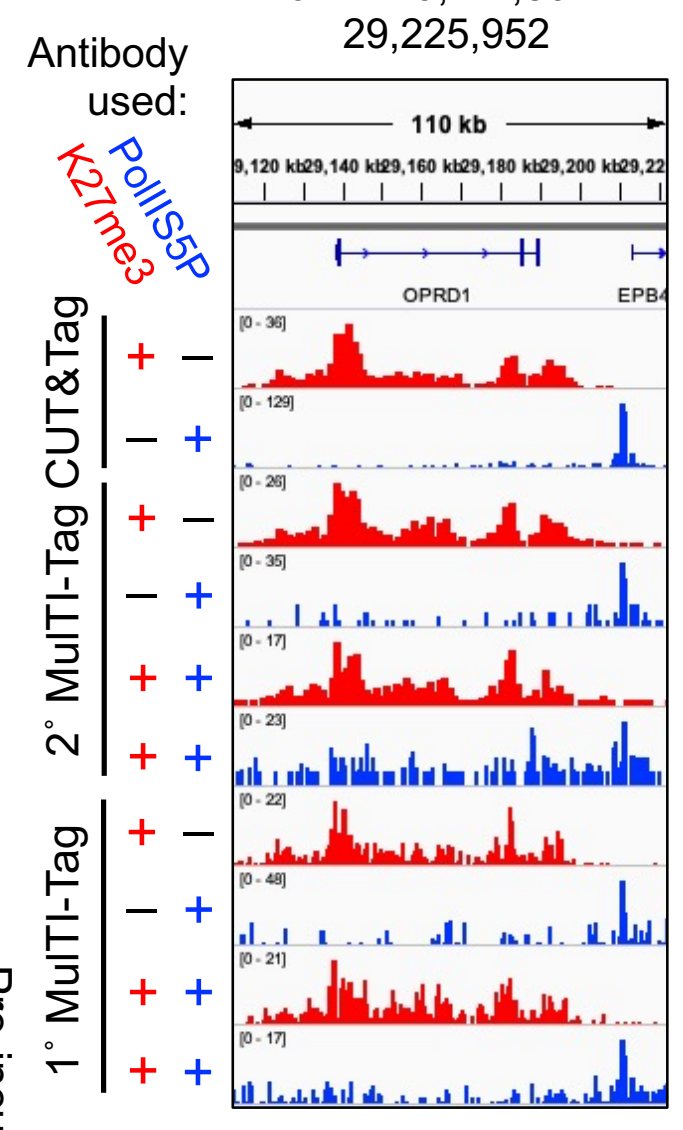

d Covalent conjugation<smiles>CC(C)(C)C(C)(C)C(C)(C)C</smiles>

$1^{\circ}$ antibody

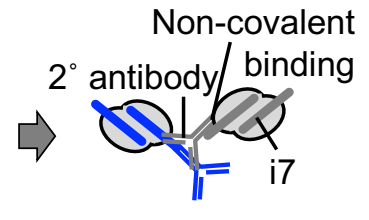

$$
\frac{\text { CUT\&Tag }}{\text { K27 K36 }} \frac{\text { MulTI-Tag }}{\text { K27 K36 } 36 \text { K27 K36 }}
$$

1

700

ㅇ. 30

200

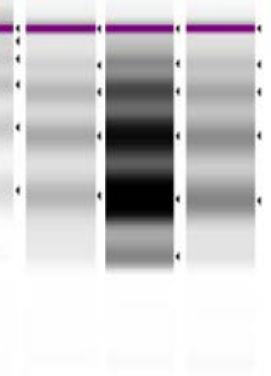


Supplementary Figure 1: a) Schematic of protocol variations tested for distinguishing CUT\&Tag targets by sequencing barcode. Top: Approaches for pairing barcodes with antibodies, either by pre-incubation of barcoded pA-Tn5 with a secondary antibody ("Preincubation", left), or covalent conjugation of barcode-containing adapters to secondary (" $2^{\circ}$ conjugate", center) or primary (" $1{ }^{\circ}$ conjugate", right) antibodies. Bottom: Approaches for tagmenting multiple targets, either in separate cells ("Individual", left), in the same cells simultaneously ("Combined", center), or in the same cells sequentially ("Sequential", right). b) Scatterplots describing the enrichment of H3K27me3 (X-axis) and PollIS5P (Yaxis) in H3K27me3 (red points) or PollIS5P (blue points) peaks for combinations of experimental conditions described in 2a. Pearson's $R^{2}$ of all data points is denoted for each of the nine protocol conditions. c) Genome browser screenshot showing individual CUT\&Tag profiles for H3K27me3 (first row) and RNA PollIS5P (second) in comparison with MulTI-Tag profiles for the same targets probed individually in different cells (third and fourth rows secondary conjugate MulTI-Tag; seventh and eighth rows primary conjugate MulTI-Tag) or sequentially in the same cells (fifth and sixth rows secondary conjugate MulTI-Tag; ninth and tenth rows primary conjugate MulTI-Tag). d) Top: Schematic of MulTI-Tag with additional CUT\&Tag step, in which $1^{\circ}$ antibody conjugates are loaded into pA-Tn5 along with free i5 adapter (left), and secondary antibody and pA-Tn5 loaded only with i7 adapter are added before tagmentation (right). Bottom: TapeStation HSD1000 trace describing DNA size and enrichment from libraries produced from CUT\&Tag (lanes 1 and 2), "standard" MulTI-Tag with conjugate-only tagmentation (3 and 4), or MulTI-Tag with a secondary CUT\&Tag step as described in methods (5 and 6), targeting H3K27me3 (1, 3, and 5) or H3K36me3 (2, 4, and 6) in K562 cells. 


\section{a}
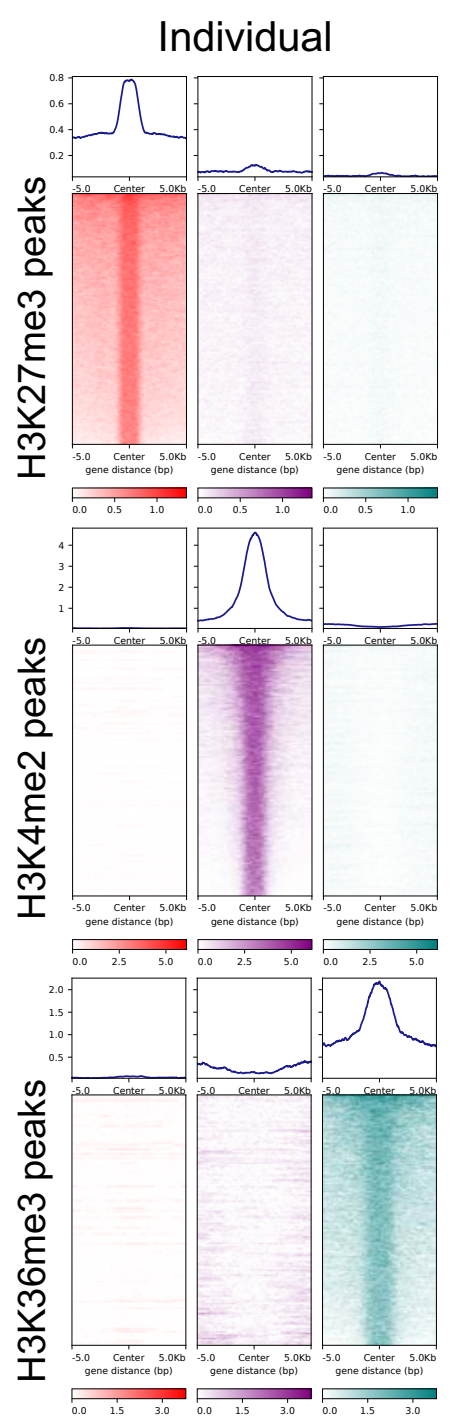

C

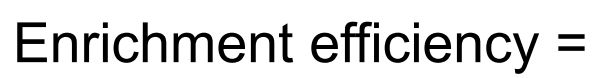

MulTI-Tag reads in target peaks/reads in all peaks CUT\&Tag reads in target peaks/reads in all peaks

\begin{tabular}{|l|l|l|l|}
\hline & H3K27me3 & H3K4me2 & H3K36me3 \\
\hline K562 & 0.5325151 & 0.5870659 & 0.8923193 \\
\hline H1 & 0.7886033 & 0.6160182 & 1.107574 \\
\hline
\end{tabular}

b
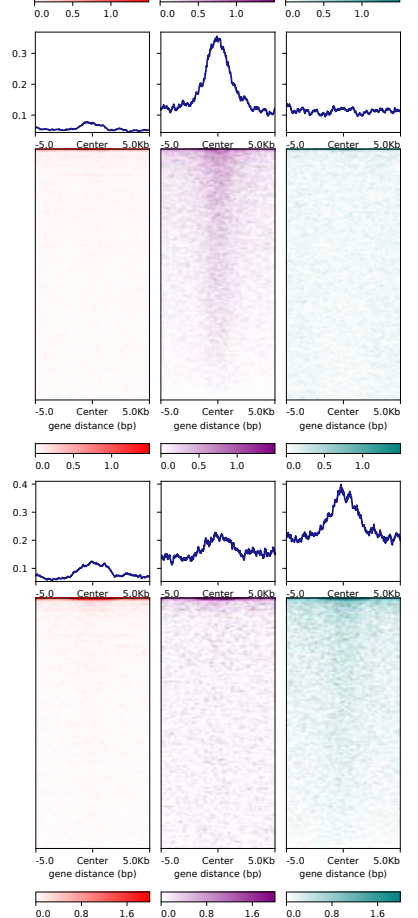

\section{Supplementary Figure 2}
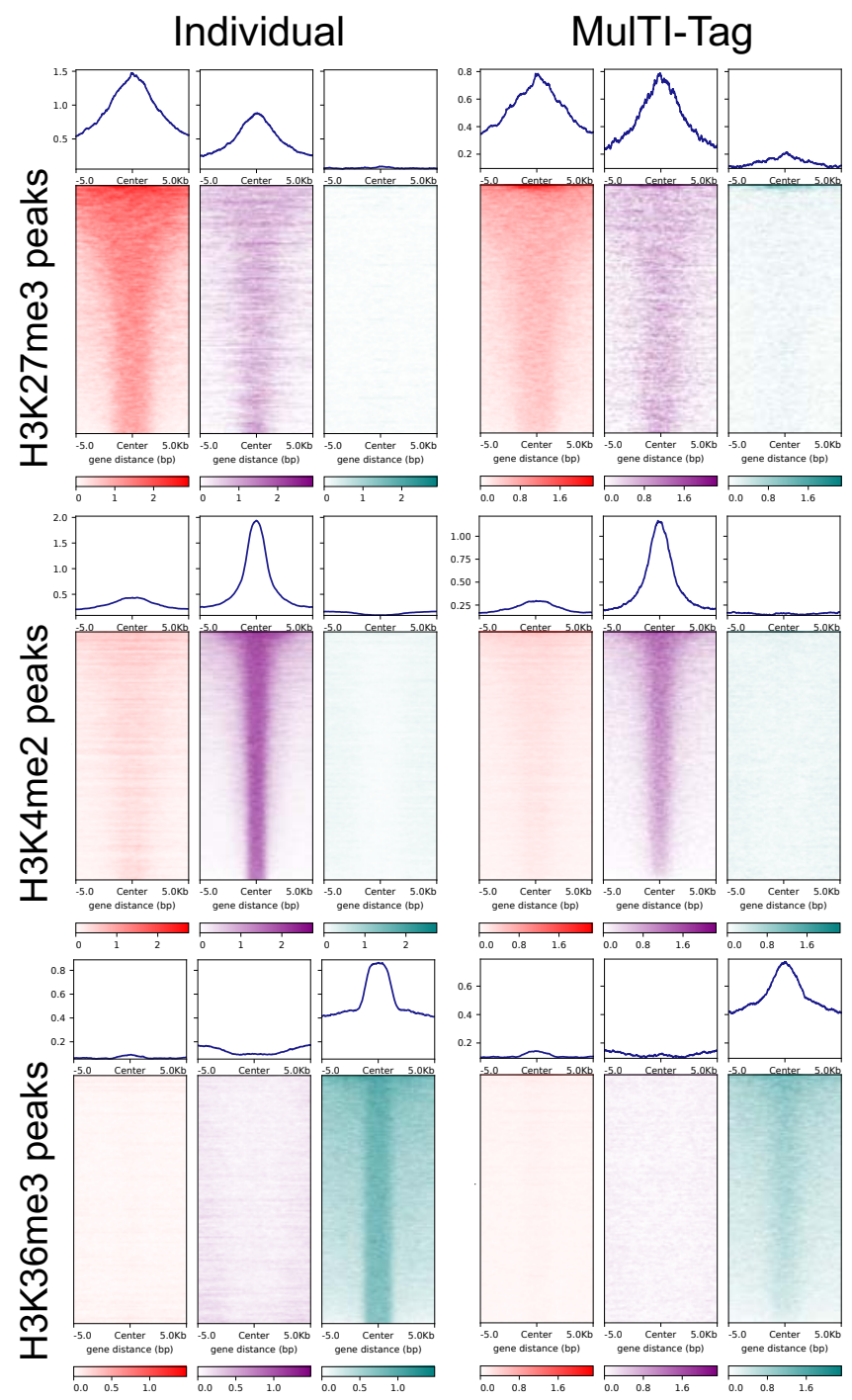

d

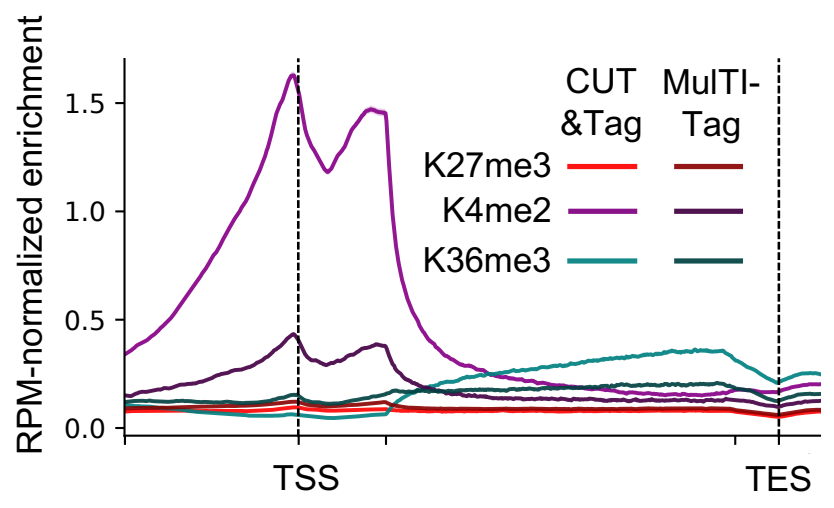


Supplementary Figure 2: a) Heatmaps describing the enrichment of H3K27me3 (red), H3K4me2 (purple), or H3K36me3 (teal) signal from K562 cell MulTI-Tag profiles using single antibodies (left) or three antibodies sequentially (right) in H3K27me3 (top), H3K4me2 (middle), or H3K36me3 (bottom) peaks. b) Heatmaps as in a) from H1 hESC MulTI-Tag experiments. c) Enrichment efficiency of MulTI-Tag vs. CUT\&Tag in peaks from cell types as denoted. d) Normalized CUT\&Tag (light colors) and MulTI-Tag (dark colors) enrichment of $\mathrm{H} 3 \mathrm{~K} 27 \mathrm{me} 3, \mathrm{H} 3 \mathrm{~K} 4 \mathrm{me} 2$, and $\mathrm{H} 3 \mathrm{~K} 36 \mathrm{me} 3$ across genes in $\mathrm{K} 562$ cells. 


\section{Supplementary Figure 3}

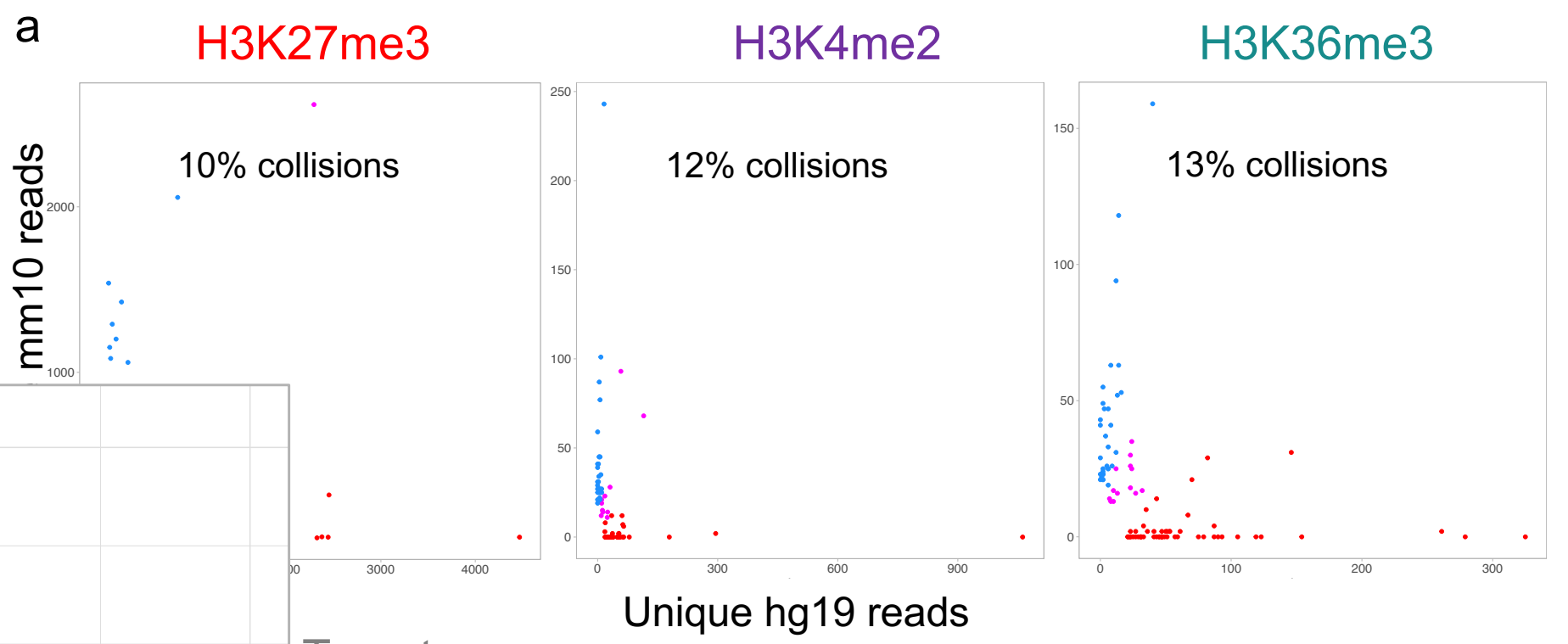

\section{Three Targets}

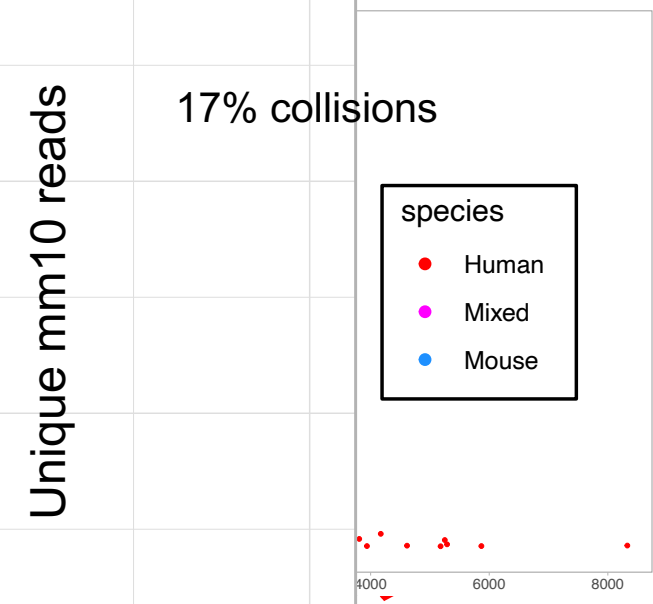

Unique hg19 reads

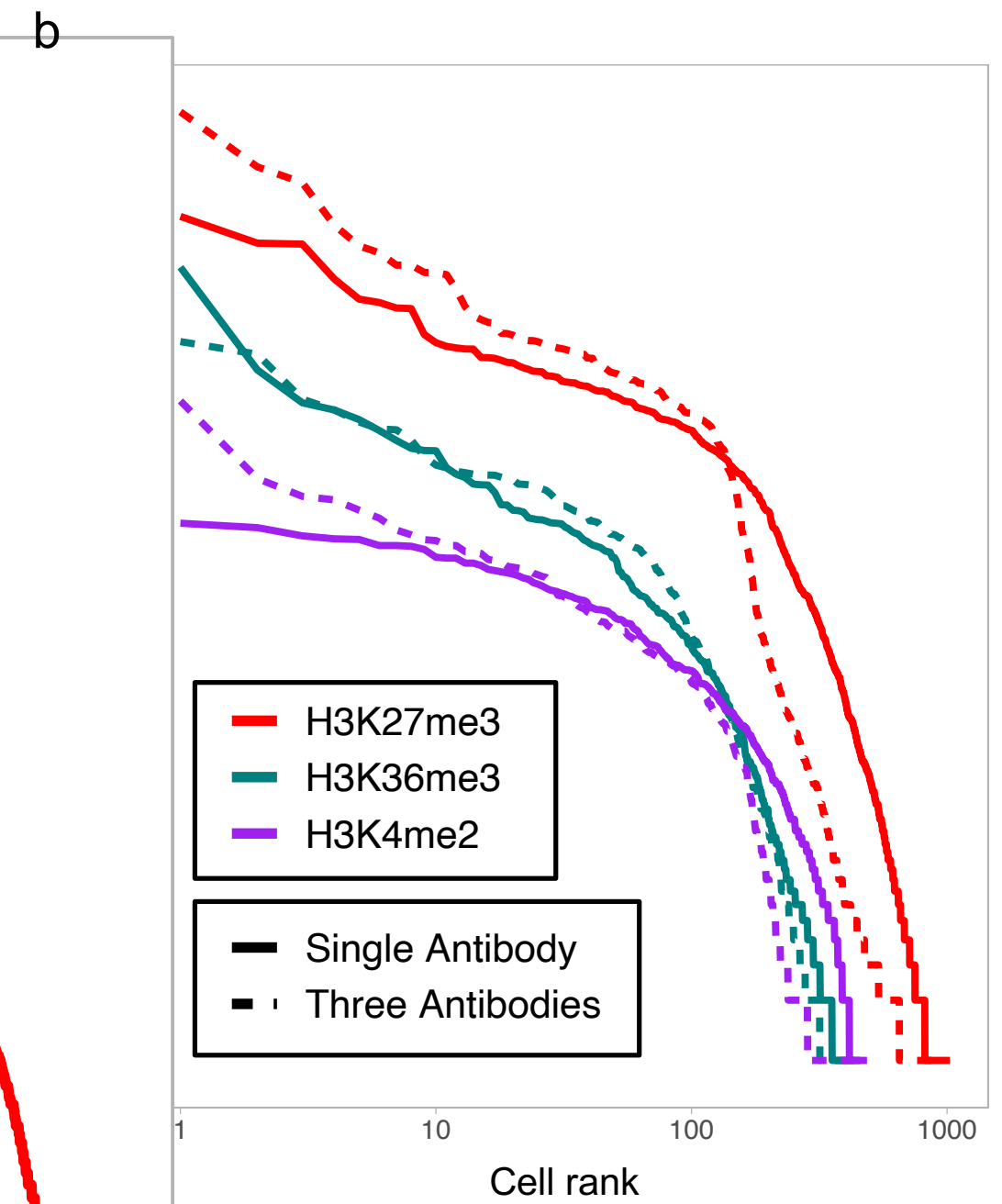


Supplementary Figure 3: a) Barnyard plots describing the number of unique fragments exclusively mapping to the hg19 genome build (X-axis) vs. mm10 (Y-axis) in the top 100 cells for each of the denoted experiments. Points are colored by the cell identity as human (red; $>70 \%$ of unique reads mapping to hg19), mouse (blue; $>70 \%$ mapping to $\mathrm{mm} 10$ ), or mixed (magenta; $<70 \%$ mapping to either), and collision rate, defined as the percentage of cells classified as "mixed", is denoted for each experiment. b) Knee plot describing unique fragments per cell (Y-axis) for cells ranked by the number of unique fragments per cell (X-axis) from MulTI-Tag experiments profiling H3K27me3 (red), H3K4me2 (purple), or H3K36me3 (teal) individually (solid line) or together (dashed line) in MulTI-Tag experiments. 
a
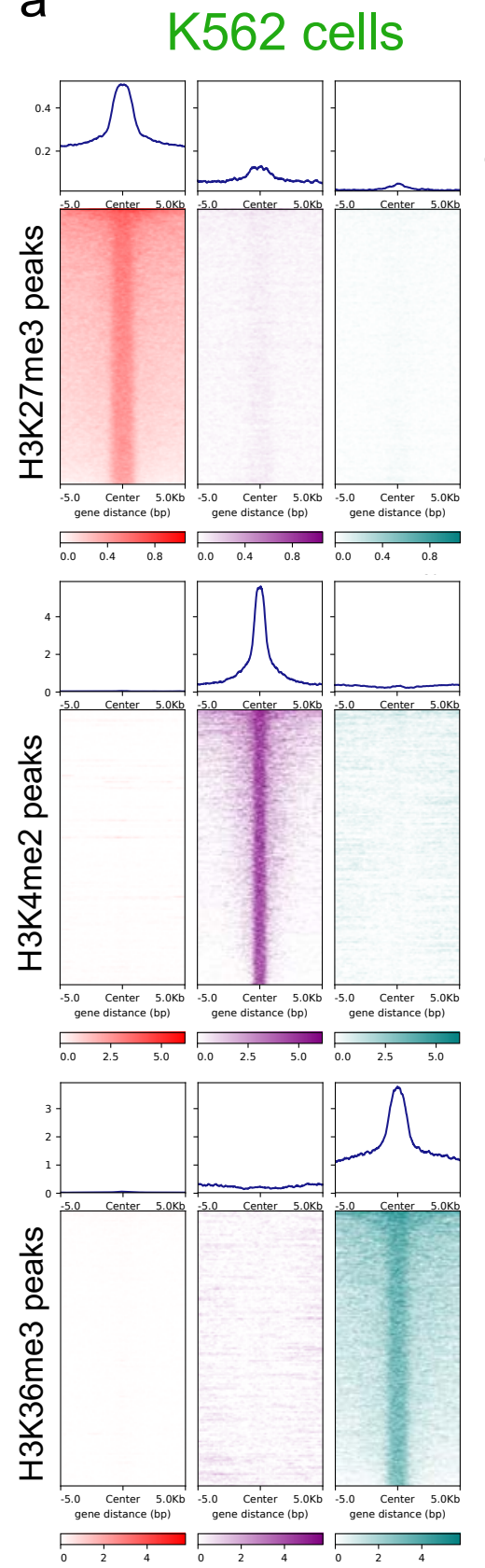

b

H1 hESCS
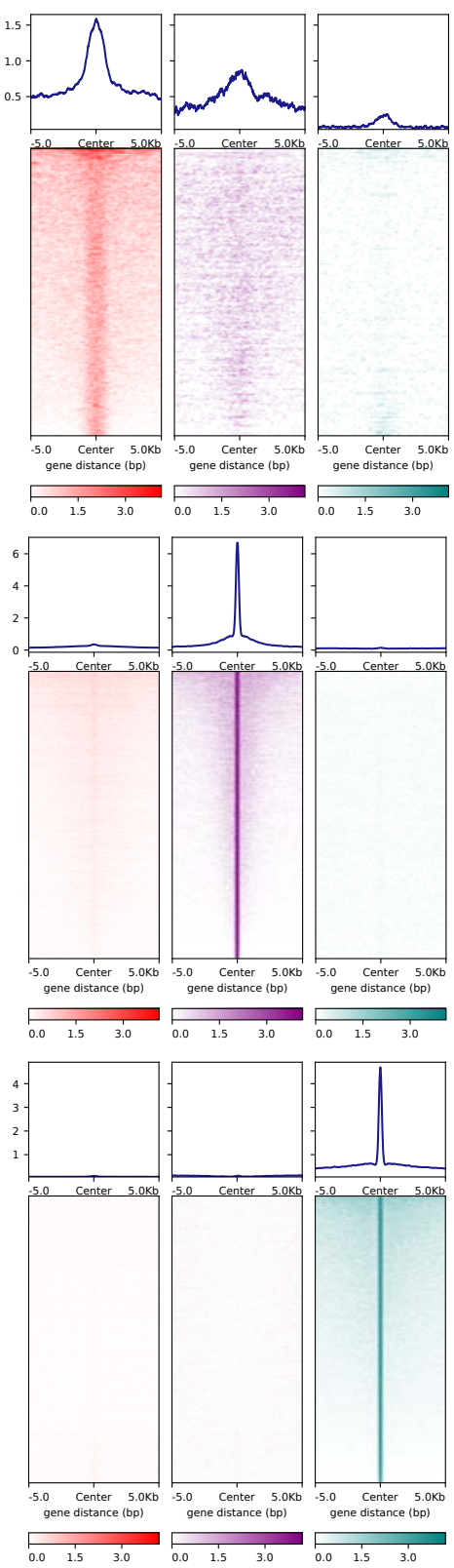

\section{Supplementary Figure 4}

C Cell type

$\square$ H1

Feature identity

$\square$ H3K27me3 $\square$ H3K4me2 $\square$ H3K36me3

K562 $0 \quad 4.328$
Normalized enrichment

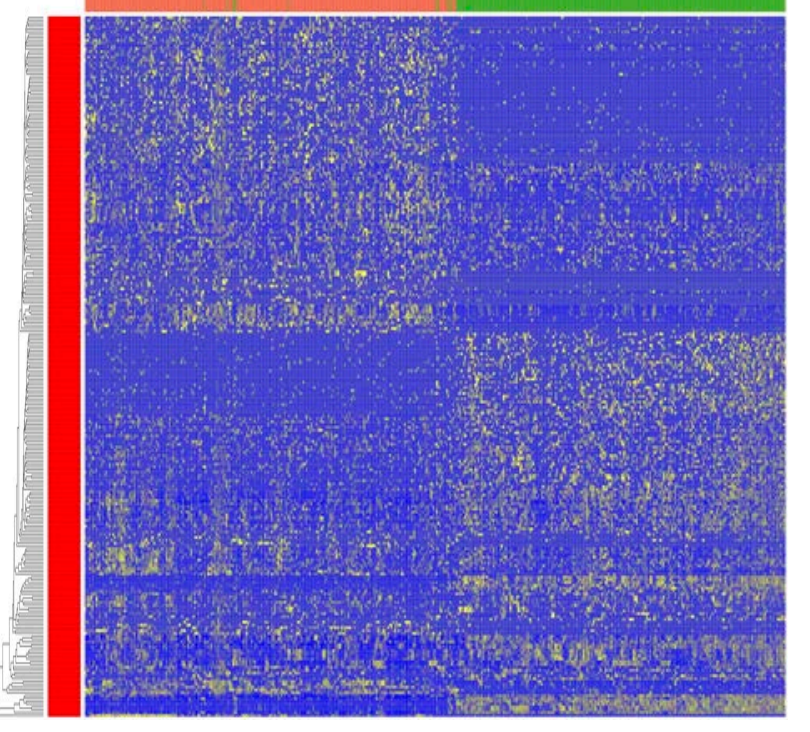

d

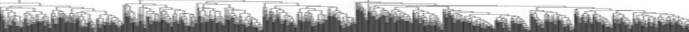


Supplementary Figure 4: a) Heatmaps describing the enrichment of H3K27me3 (red), H3K4me2 (purple), or H3K36me3 (teal) signal from aggregate single cell MulTI-Tag profiles from K562 cellsin H3K27me3 (top), H3K4me2 (middle), or H3K36me3 (bottom) peaks. b) Heatmaps as in a) for $\mathrm{H} 1$ hESC MulTI-Tag experiments. c) Hierarchically clustered heatmap describing MulTI-Tag enrichment from single cells (columns) in the 248 most variable defined features (rows) in the experiment. Color coding above columns indicates cell type, and color coding to the left of rows indicates feature identity. d) Hierarchically clustered heatmap describing MulTI-Tag enrichment from single cells (columns) in 164 features (rows) defined as most variable relative to other features of the same target identity in the experiment. Color coding above columns indicates cell type, and color coding to the left of rows indicates feature identity. 
a

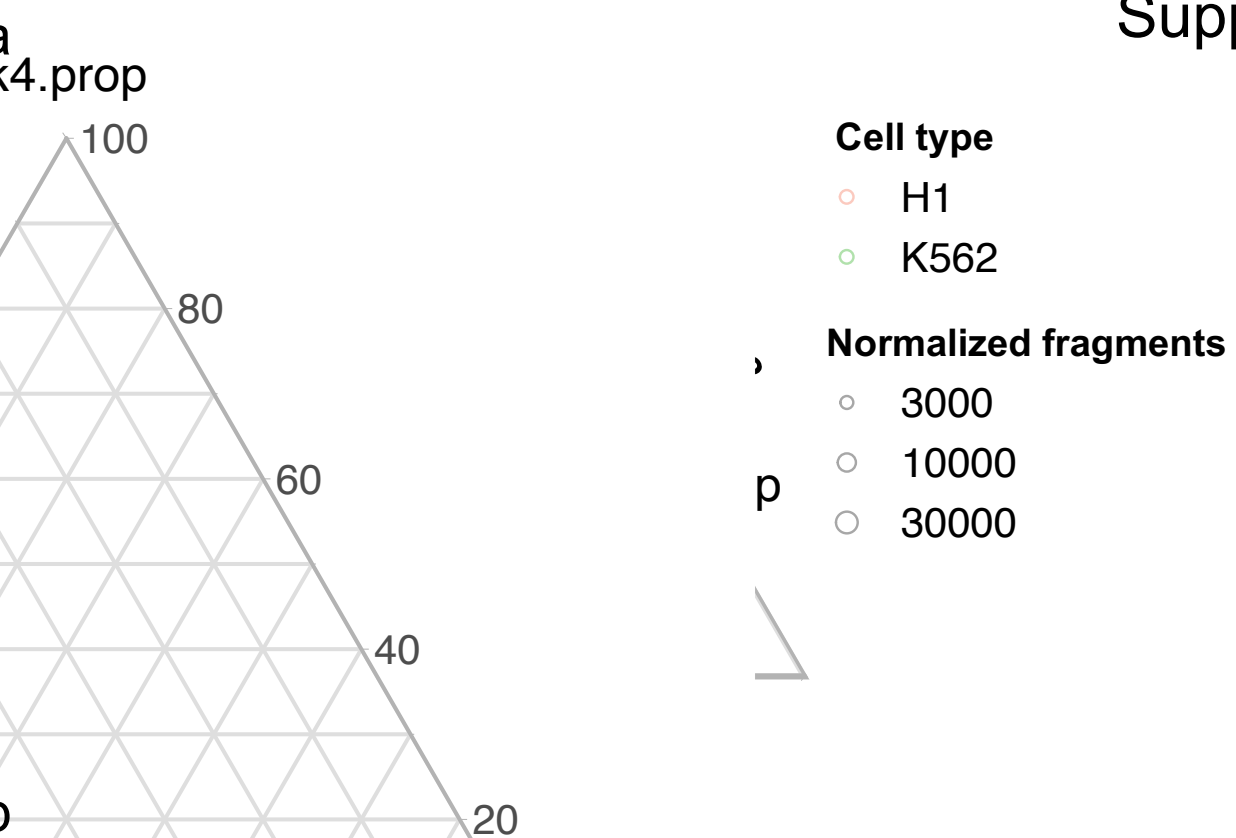

\section{Supplementary Figure 5}

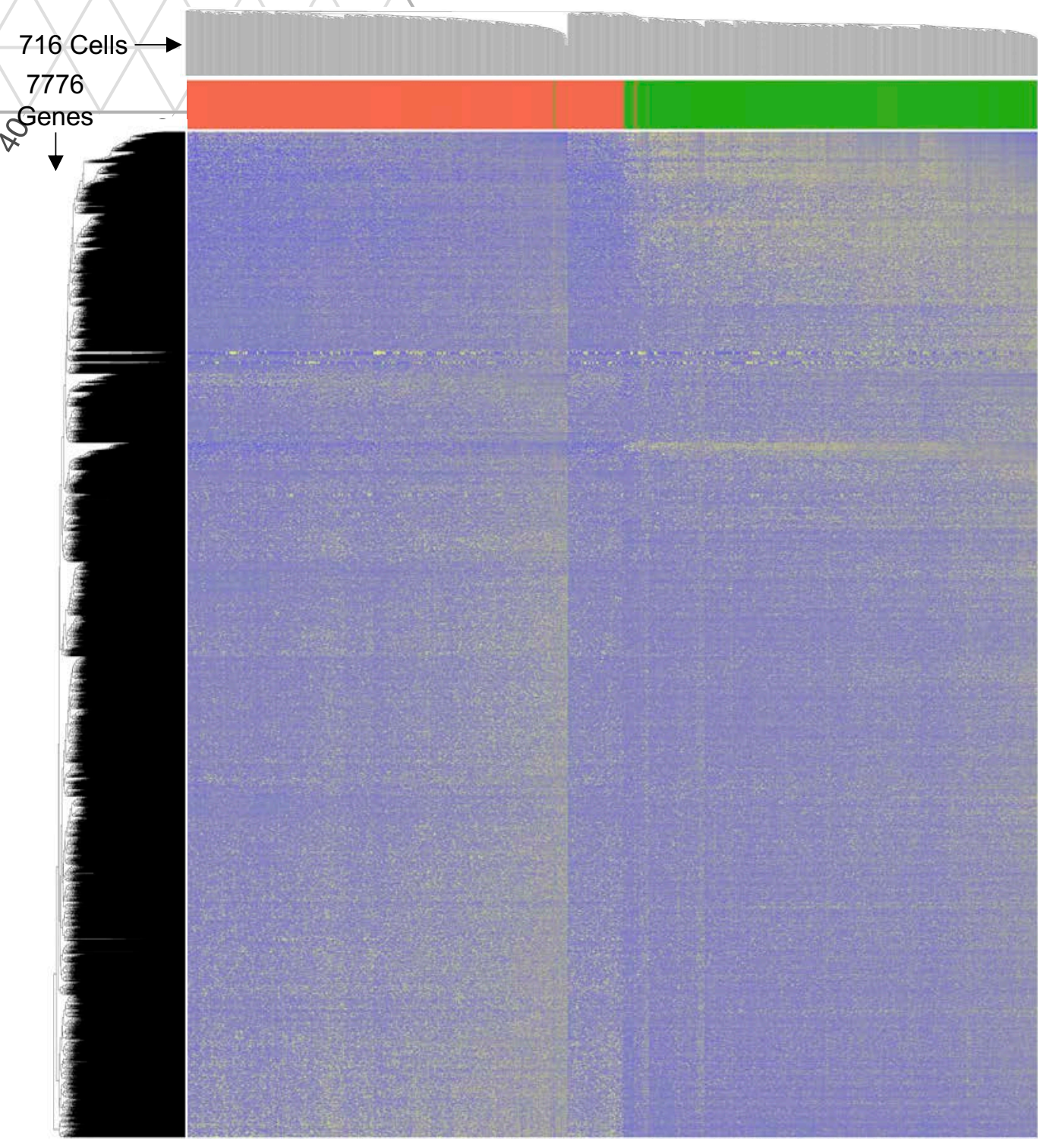

Cell type

$\square$ H1 $\square$ K562

Normalized enrichment

0

2.185 
Supplementary Figure 5: a) Ternary plot describing the percentage of fragments originating from $\mathrm{H} 3 \mathrm{~K} 27 \mathrm{me} 3$ (left axis), $\mathrm{H} 3 \mathrm{~K} 4 \mathrm{me} 2$ (right axis), or H3K36me3 (bottom axis) in each cell (open circles) in single cell MulTI-Tag. Cell identity is denoted by circle color, and total fragment count is denoted by circle size. b) Hierarchically clustered heatmap describing MulTI-Tag enrichment for all three targets from single cells (columns) in 7776 genes (rows) that are in the top $40 \%$ of genes by total fragments mapped. Color coding above columns indicates cell type. 
a

ENCODE ChIP-seq
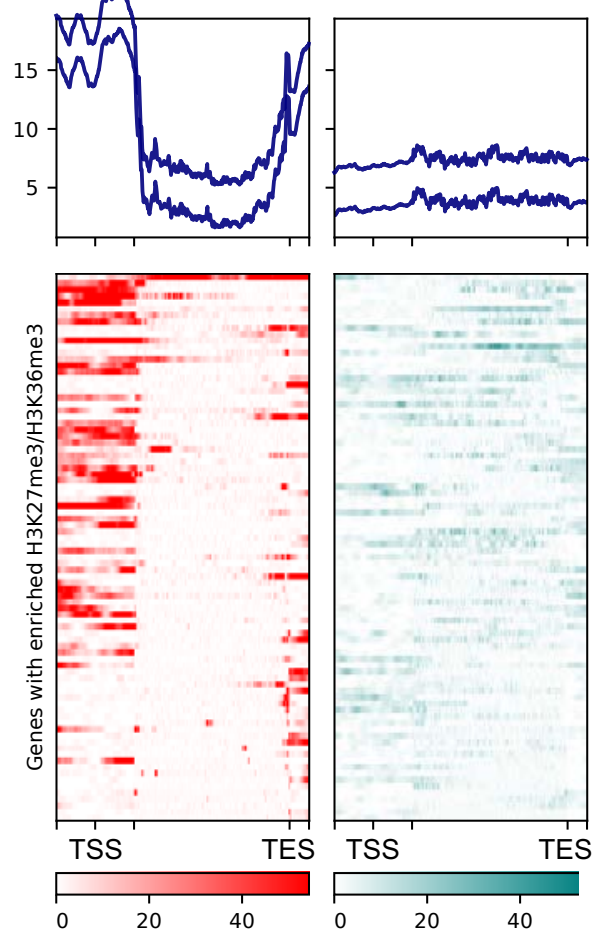

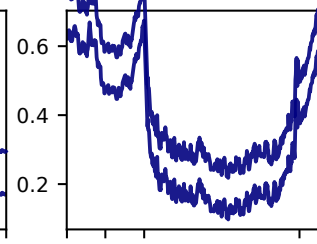

MulTI-Tag
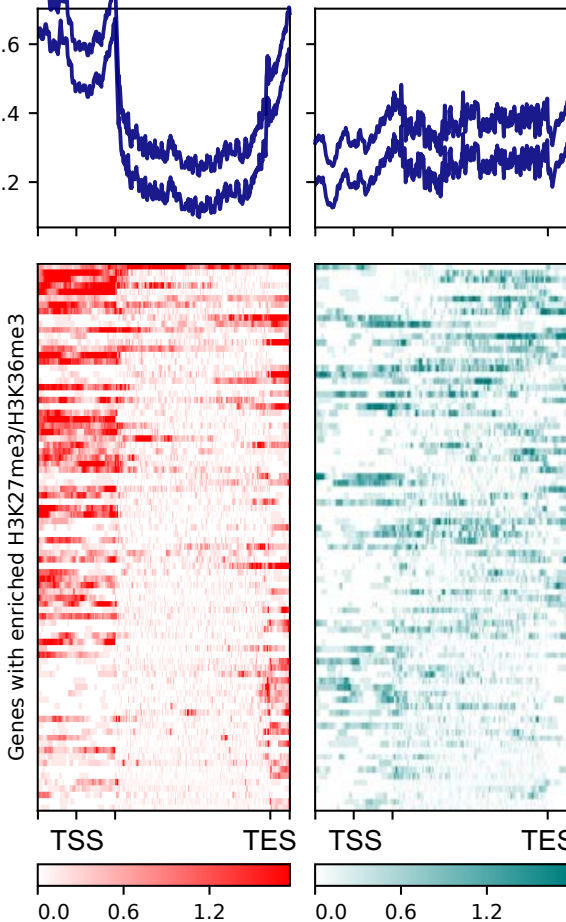

H3K27me3

H3K36me3

\section{Supplementary Figure 6}

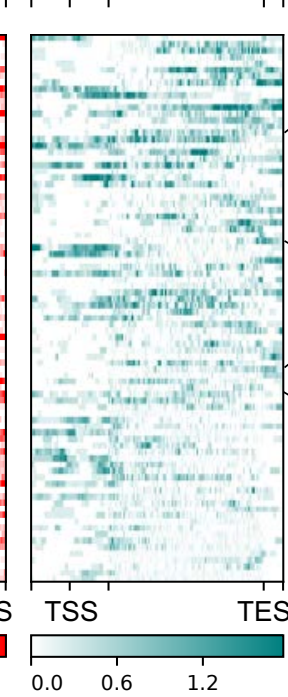

\section{PCSK9}

TGFB1

BMP6

$\mathrm{PTCH} 1$

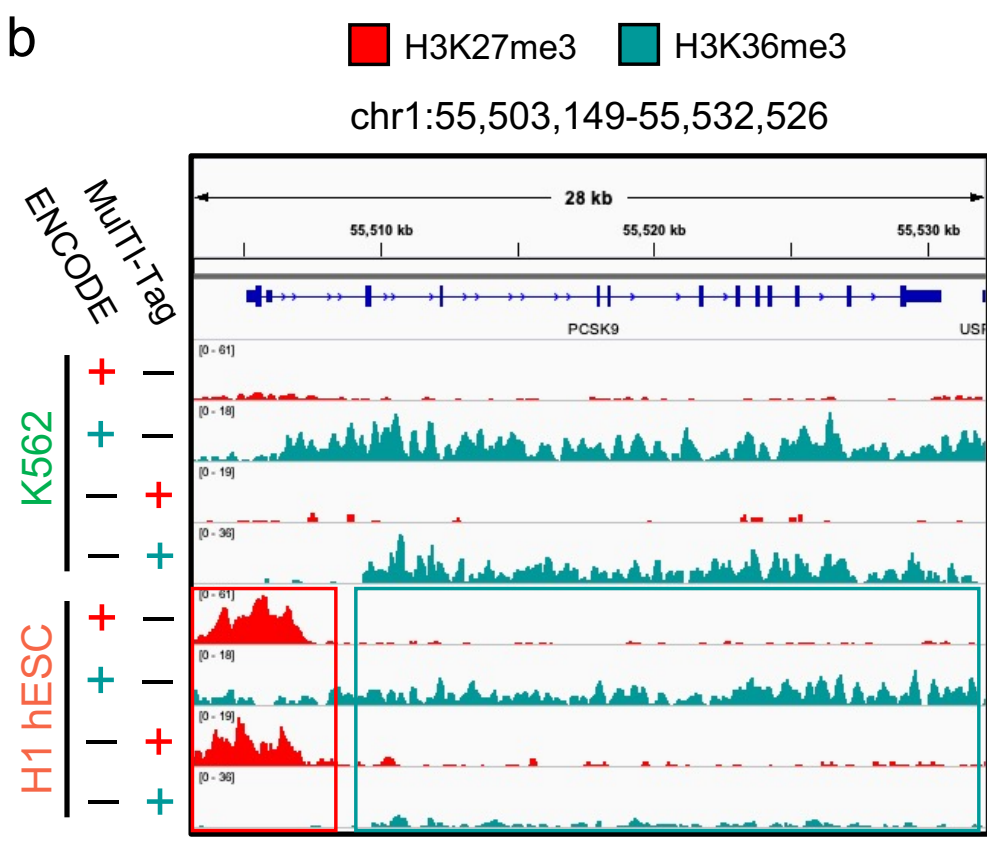

chr9:98,193,264-98,291,247

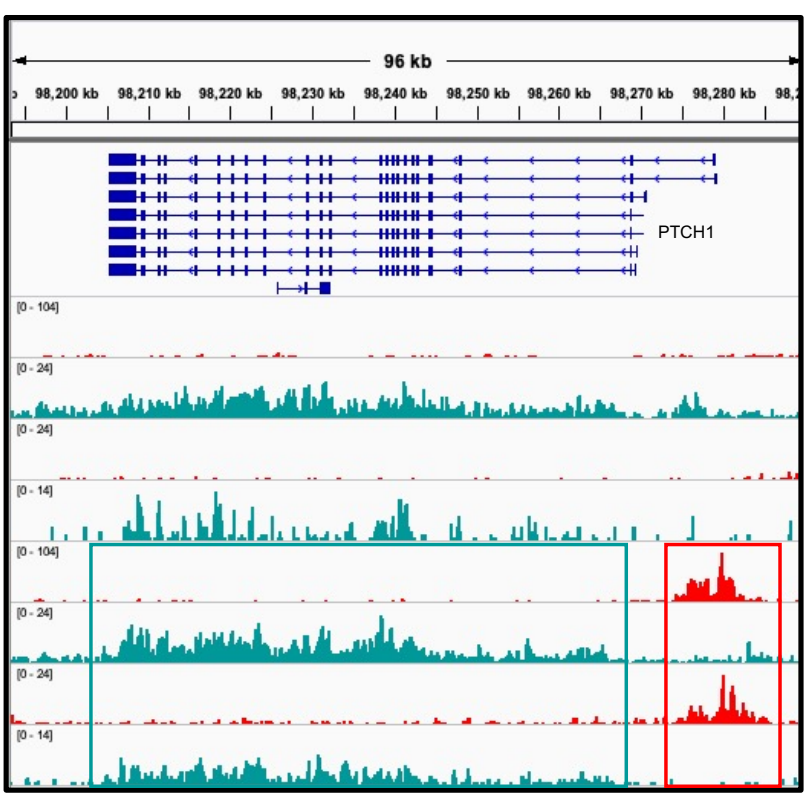


Supplementary Figure 6: a) Heatmaps describing the enrichment of $\mathrm{H} 3 \mathrm{~K} 27 \mathrm{me} 3$ (red) and H3K36me3 (teal) signal from ENCODE ChIP-seq (left) or bulk MulTI-Tag (right) in $\mathrm{H} 1 \mathrm{hESC}$ in 86 genes for which 1) a MulTI-Tag H3K27me3 peak overlapped a $2 \mathrm{~kb}$ window surrounding the TSS, and 2) a MulTI-Tag H3K36me3 peak overlapped the gene body. Selected genes of interest, including those involved in metabolic and developmental signaling, are highlighted at right. b) Genome browser screenshots showing H3K27me3 (red) and H3K36me3 (teal) enrichment from ENCODE ChIP-seq (rows 1, 2, 5, and 6) or bulk MulTI-Tag (rows 3, 4, 7, and 8) in K562 cells (rows 1-4) or H1 hESCs (rows 5-8) at the PCSK9 (left) and PTCH1 (right) genes. Colored boxes indicate co-enrichment of $\mathrm{H} 3 \mathrm{~K} 27 \mathrm{me} 3$ and $\mathrm{H} 3 \mathrm{~K} 36 \mathrm{me} 3$ in the same gene in $\mathrm{H} 1 \mathrm{hESC}$. Gene model for PTCH1 is expanded to visualize alternative promoter structure. 